Research Article

\title{
The Mutual Inhibition of FoxO1 and SREBP-1c Regulated the Progression of Hepatoblastoma by Regulating Fatty Acid Metabolism
}

\author{
Yu Hu, Hongyan Zai, Wei Jiang, Zhenglin Ou, Yuanbing Yao, and Qin Zhu \\ Department of General Surgery, Xiangya Hospital, Central South University, Changsha, 410008, China \\ Correspondence should be addressed to Qin Zhu; zhuqinn08@163.com
}

Received 22 April 2021; Revised 22 July 2021; Accepted 25 August 2021; Published 8 September 2021

Academic Editor: Shuai Chen

Copyright (C) $2021 \mathrm{Yu} \mathrm{Hu}$ et al. This is an open access article distributed under the Creative Commons Attribution License, which permits unrestricted use, distribution, and reproduction in any medium, provided the original work is properly cited.

\begin{abstract}
Background. Hepatoblastoma (HB) is the most common liver malignancy in pediatrics, but the treatment for this disease is minimal. This study is aimed at exploring the effect of FoxO1 and SREBP-1c on $\mathrm{HB}$ and their mechanism. Methods. FoxO1, SREBP-1c, FASN, ACLY, ACC, and MAGL expressions in tissue samples were detected by RT-qPCR and WB. IHC was utilized to measure FASN content. Overexpression and knockdown of FoxO1 and sSREBP-1c were performed on Huh-6 cells. Cell proliferation, migration, and invasion were examined by CCK8, scratch, and transwell assay. ELISA was performed to test the ATP, FAO, NEFA, and Acetyl-CoA contents. ChIP was used to detect the interaction between SREBP-1c protein and the FoxO1 gene. In vivo tumorigenesis was conducted on mice. The morphology of tumor tissue sections was observed by HE staining. Results. FoxO1 expression was downregulated in HB tissue, while the expressions of SREBP-1c, FASN, ACLY, ACC, and MAGL were upregulated. In Huh-6 cells and mouse tumor tissues, FoxO1 knockdown resulted in increased cell proliferation, migration, and invasion and active fatty acid metabolism. On the contrary, after the knockdown of SREBP-1c, cell proliferation, migration, and invasion were weakened, and fatty acid metabolism was significantly reduced. SREBP-1c interacted with the promoter of the FoxO1 gene. When FoxO1 was knocked down, the tumor tissue was more closely packed. After the knockdown of the SREBP-1c gene, the structure of tumor cells was deformed. Conclusion. FoxO1 and SREBP-1c inhibited each other in HB, leading to the increase of intracellular fatty acid metabolism, and ultimately facilitated the development of HB.
\end{abstract}

\section{Introduction}

Hepatoblastoma (HB) is a pediatric tumor caused by hepatic progenitors or hepatoblasts. It is the most common liver malignant tumor in pediatrics. Its annual incidence is 1.5 cases per million, accounting for about $1 \%$ of all childhood cancers [1]. The primary treatment for HB is surgical resection, but about $60 \%$ of the tumors are unresectable at the onset, so the therapeutic effect is minimal [2]. Therefore, there is an urgent need to explore the pathogenesis of $\mathrm{HB}$ and develop new therapeutic targets to improve the clinical outcome of HB patients. One of the characteristics of cancer cells is reprogramming fatty acid metabolism [3]. Variation in lipid metabolisms, such as increased fatty acid uptake, de novo lipogenesis, is closely related to the generation of cancer cells [4]. The expression and activity of enzymes involved in lipid metabolism are significantly increased in many cancer cells, such as fatty acid synthase (FASN) and Acetyl-coenzyme A carboxylase (ACC) [5]. FASN plays a crucial role in lipid metabolism and has become an attractive target in clinical cancer treatment [6]. However, the mechanism of lipid metabolism in $\mathrm{HB}$ is still unclear.

The forkhead box-O1 (FoxO1) is a central regulator of metazoan physiology and plays a role in cell cycle, proliferation, apoptosis, autophagy, stress resistance, DNA repair, tumor inhibition, metabolism, and other cellular activities [7]. FoxO1 is tightly regulated by modifying its mRNA and protein, and its expression is regulated by nutritional signals in the environment [8]. Dysfunction of the FoxO1 pathway leads to various metabolic diseases, including diabetes, 
obesity, nonalcoholic fatty liver disease, and atherosclerosis [8]. FoxO1 is also thought to inhibit the development of osteosarcoma, but the mechanism of its inhibitory effect is not precise [9]. FoxO1 also plays a vital role in fat metabolism. It is reported that FoxO1 can slow down lipid deposition in the liver caused by stress response [10].

Sterol regulatory element-binding proteins are a class of transcription factors that regulate lipid homeostasis by controlling the synthesis of cholesterol, fatty acids, triglycerides, and phospholipids [11]. Among them, sterol regulatory element-binding protein-1c (SREBP-1c) is derived from the SREBP-1c gene on chromosome 17 and mainly regulates the synthesis of fatty acids and triglycerides [12]. It is an essential link between oncogenic signals and tumor metabolism [13]. Activation of SREBP-1c causes upregulation of FASN, enhances fatty acid metabolism, and theoretically promotes cancer development [14]. Geng et al. believed that SREBP-1c-driven lipid metabolism could be targeted to treat glioblastoma [15]. The regulatory pathway of FoxO1 and SREBP-1c in endometrial cancer was established [16]. FoxO1 inhibited insulin-induced SREBP-1c promoter activity in goat mammary epithelial cells and the transcription of SREBP-1c by the liver $\mathrm{X}$ receptor response element and SREBP response element on the SREBP-1c promoter [17]. However, it is still elusive whether FoxO1 and SREBP-1c play a role in regulating fatty acid metabolism in $\mathrm{HB}$.

Derive from the above background, we wanted to explore the effect of FoxO1 and SREBP-1c on HB cells and study the mechanism of fatty acid metabolism in HB. Therefore, we collected clinical samples of $\mathrm{HB}$ and paracancerous tissues, purchased various $\mathrm{HB}$ cell lines, and conducted in vitro and in vivo experiments. This study contributes to our further understanding of the pathophysiology of $\mathrm{HB}$ and is expected to provide a new approach for the clinical treatment of $\mathrm{HB}$ patients.

\section{Materials and Methods}

2.1. Tissues and Cells. Clinical HB and paracancerous tissue samples were collected from Xiangya Hospital and divided into HB and control groups, with 5 samples in each group. The Human Research Ethics Committee of Xiangya Hospital approved this study (No. AF/SQ202104798). HB cells including HepG2 (bio-105877), HB611 (bio-73286), Huh-6 (bio-73060), and human normal liver cell WRL68 (bio53604) were purchased from Biobw and cultured in DMEM medium (D5796, Sigma) containing 10\% fetal bovine serum (\#10099141, Gibco) at $37^{\circ} \mathrm{C}$ and $5 \% \mathrm{CO}_{2}$. In order to investigate the effect of FoxO1, Huh-6 cells were randomly divided into 5 groups (the control, the oe-NC, the oe-FoxO1, the si-NC, and the si-FoxO1 groups). In the second majority of the study, Huh- 6 cells were divided into 5 groups (control, si-NC, si-FoxO1, si-SREBP-1c, and si-FoxO1+si-SREBP-1c groups) to study the effect of SREBP-1c.

2.2. Vector Recombination and Cell Transfection. The siFoxO1 and si-SREBP-1c vectors were obtained by integrating the shRNA sequence targeting human FoxO1 or SREBP-1c into a psi-LVRU6MP lentivirus vector (GeneCo- poeia). The human FoxO1 cDNA sequence was inserted into the $\mathrm{pCDH}-\mathrm{CMV}-\mathrm{MCS}-\mathrm{EF} 1 \mathrm{a}$ vector (Epoch Life Science Inc) to get the oe-FoxO1 vector. The empty psi-LVRU6MP lentivirus vector was used as the si-FoxO1 vector. The empty pCDH-CMV-MCS-EF1a vector was the oe-FoxO1 vector. Then, the constructed vector was transfected into $293 \mathrm{~T}$ cells (HEK293T, Procell) to produce lentiviral solutions. These lentiviral solutions were transfected into Huh-6 cells with the assistance of $8 \mu \mathrm{g} / \mathrm{mL}$ of Polybrene (\#H9268, SigmaAldrich) [18]. Forty-eight hours after transfection [19], the cells were further examined.

2.3. Real-Time Quantitative Polymerase Chain Reaction (RT$q P C R)$. The trizol method was used to extract total RNA from cells and tissues. cDNA was obtained by reverse transcription using an mRNA reverse transcription kit (\#CW2569, Cowin Bio). Primer sequences of FoxO1, SREBP-1c, FASN, ATP-citric acid lyase (ACLY), ACC, monoacylglycerol lipase (MAGL), and $\beta$-actin were designed (Table 1). Shanghai Sangon Biotech synthesized the primers. Fluorescent dye was added to prepare the PCR reaction system. DNA amplification was performed by a fluorescent quantitative PCR apparatus (PIKOREAL96, Thermo). The amplification and fusion curves of each gene were obtained by real-time monitoring of fluorescence signals. $\beta$-Actin was used as an internal reference. The relative expression of genes was calculated using the $2^{-\Delta \Delta \mathrm{CT}}$ method.

2.4. Western Blot (WB). Total protein of cells and tissues was extracted with RIPA lysate (\#P0013B, Beyotime). The mixture was bathed in water for $5 \mathrm{~min}$ after the protein supernatant was thoroughly mixed with the loading buffer. Then, the protein samples were isolated on gel and electrophoresis at a constant pressure of $75 \mathrm{~V}$ for $130 \mathrm{~min}$. After electrophoresis, the target protein was transferred to the nitrocellulose membrane. The membranes were sealed and then incubated with primary antibodies FASN (1:2000, 10624-1-AP, Proteintech), ACLY (1:10000, 67166-1-Ig, Proteintech), ACC (1:4000, 21923-1AP, Proteintech), MAGL (1:5000, ab124796, Abcam), and $\beta$-actin $(1: 5000,60008-1-\mathrm{Ig}$, Proteintech) for $90 \mathrm{~min}$. After incubation, the membranes were washed with PBST. The membranes and secondary antibody HRP goat anti-mouse IgG (SA00001-1, 1:5000, Proteintech) or HRP goat antirabbit IgG (SA00001-2, 1:6000, Proteintech) were then incubated for $90 \mathrm{~min}$. Finally, the strips on the membranes were visualized using SuperECL Plus hypersensitive luminescence solution (K-12045-D50, Advansta). Grayscale values for all stripes were determined by Photoshop 2019. $\beta$-Actin was used as an internal parameter. The expression of the protein was expressed by the ratio of the grayscale value of the target protein to that of the reference protein.

2.5. Immunohistochemistry (IHC). IHC detected the expression of FASN in tissues. Paraffin sections of $\mathrm{HB}$ and paracancerous tissues were made. After the sections were deparaffinized and rehydrated, they were heated in a microwave oven to repair the antigen. The endoenzymes were inactivated by adding $1 \%$ periodate acid to the sections. Then, sections and the anti-FASN antibody (10624-1-AP, 
TABLE 1: RT-qPCR primer sequences.

\begin{tabular}{|c|c|c|}
\hline Gene & Sequences $\left(5^{\prime}-3^{\prime}\right)$ & Product length $(\mathrm{bp})$ \\
\hline \multirow{2}{*}{ FoxO1 } & F: АСТTCATCTCATTCTCССТTCTGC & \multirow{2}{*}{199} \\
\hline & R: GCACAACTTACAGCTGGTTTTCAA & \\
\hline \multirow{2}{*}{$A C L Y$} & F: CCTCAGCCATCCAGAATCGG & \multirow{2}{*}{194} \\
\hline & R: CTTCAGCCAGGACTTGACCC & \\
\hline \multirow{2}{*}{$S R E B P-1 c$} & F: GCTCCCTAGGAAGGGCCGTA & \multirow{2}{*}{240} \\
\hline & R: CACTCTTAGTTTTCCTTCCGTTT & \\
\hline \multirow{2}{*}{ FASN } & F: CCTGGCTGCCTACTACATCG & \multirow{2}{*}{102} \\
\hline & R: CACATTTCAAAGGCCACGCA & \\
\hline \multirow{2}{*}{$A C C$} & F: CTCTTGGCCTTTTCCCGGTC & \multirow{2}{*}{228} \\
\hline & R: GTTATCCCCAAACCCAGGCA & \\
\hline \multirow{2}{*}{$M A G L$} & F: TCCAGCATGCCAGAGGAAAG & \multirow{2}{*}{142} \\
\hline & R: TGGGACACAAAGATGAGGGC & \\
\hline \multirow{2}{*}{$\beta$-Actin } & F: ACATCCGTAAAGACCTCTATGCC & \multirow{2}{*}{224} \\
\hline & R: AGCACAGCCTGGATAGCAAC & \\
\hline
\end{tabular}

TABLe 2: FoxO1 primer sequences.

\begin{tabular}{lcc}
\hline Primer & Sequences $\left(5^{\prime}-3^{\prime}\right)$ & Product length $(\mathrm{bp})$ \\
\hline 1 & F: CAGAACCCCATGGCTAAGGTC & 153 \\
\hline 2 & R: ATCTAATCCTGGCTCATTCCT & 165 \\
\hline 3 & F: ACACTGAGGGTCCATCCCA & 125 \\
\hline 4 & F: : TGTTTTCACACTGAACTGTGCAT & 108 \\
\hline 5 & R: TGGCCGATTCACAGATCAAGA & 168 \\
\hline 6 & F: ACACTGGAAGACCTTTGCCTT & 159 \\
\hline
\end{tabular}

$1: 100$, Proteintech) were incubated overnight at $4^{\circ} \mathrm{C}$ and then incubated with the second antibody for $30 \mathrm{~min}$ the next day. The sections were rinsed with PBS solution and incubated with DAB solution (ZSGB-BIO) for $5 \mathrm{~min}$ at room temperature. Sections were re-stained with hematoxylin for $5 \mathrm{~min}$. The sections were treated with alcohol and xylene and sealed with neutral resin. Microscope (BA410T, MOTIC) was used to capture images, and the image analysis software was Image-Pro-Plus. The average IOD was calculated by the ratio of the cumulative optical density of the positive expression site to the sample area in view.

2.6. Cell Counting Kit-8 (CCK8) Assay. The cells were digested with trypsin and resuspended in a DMEM medium. Cells were seeded in a $5 \times 10^{3}$ cells/well density in a 96-well plate of $100 \mu \mathrm{L}$ per well. The plates were placed in an incubator at $37^{\circ} \mathrm{C}$ and $5 \% \mathrm{CO}_{2}$ for preculture. $10 \mu \mathrm{L}$ CCK8 solution (NU679, Dojindo) was added to each well. Cells were further incubated in the incubator for $4 \mathrm{~h}$. Bio-Tek microplate analyzer (MB-530, HEALES) was used to measure the absorbance at $450 \mathrm{~nm}$.

2.7. Scratch Assay. Trypsin was used to digest the cells in the logarithmic growth phase were digested into a single-cell suspension. Cells were seeded into a 6 -well culture plate at a density of $5 \times 10^{5}$ cells per well. The cells were cultured at $37^{\circ} \mathrm{C}$ in a $5 \% \mathrm{CO}_{2}$ incubator for about $24 \mathrm{~h}$ until covered with six-well plates. A scratch was made with a pipette tip along the transverse line behind the six-well plates. The plates were washed three times with PBS to remove 

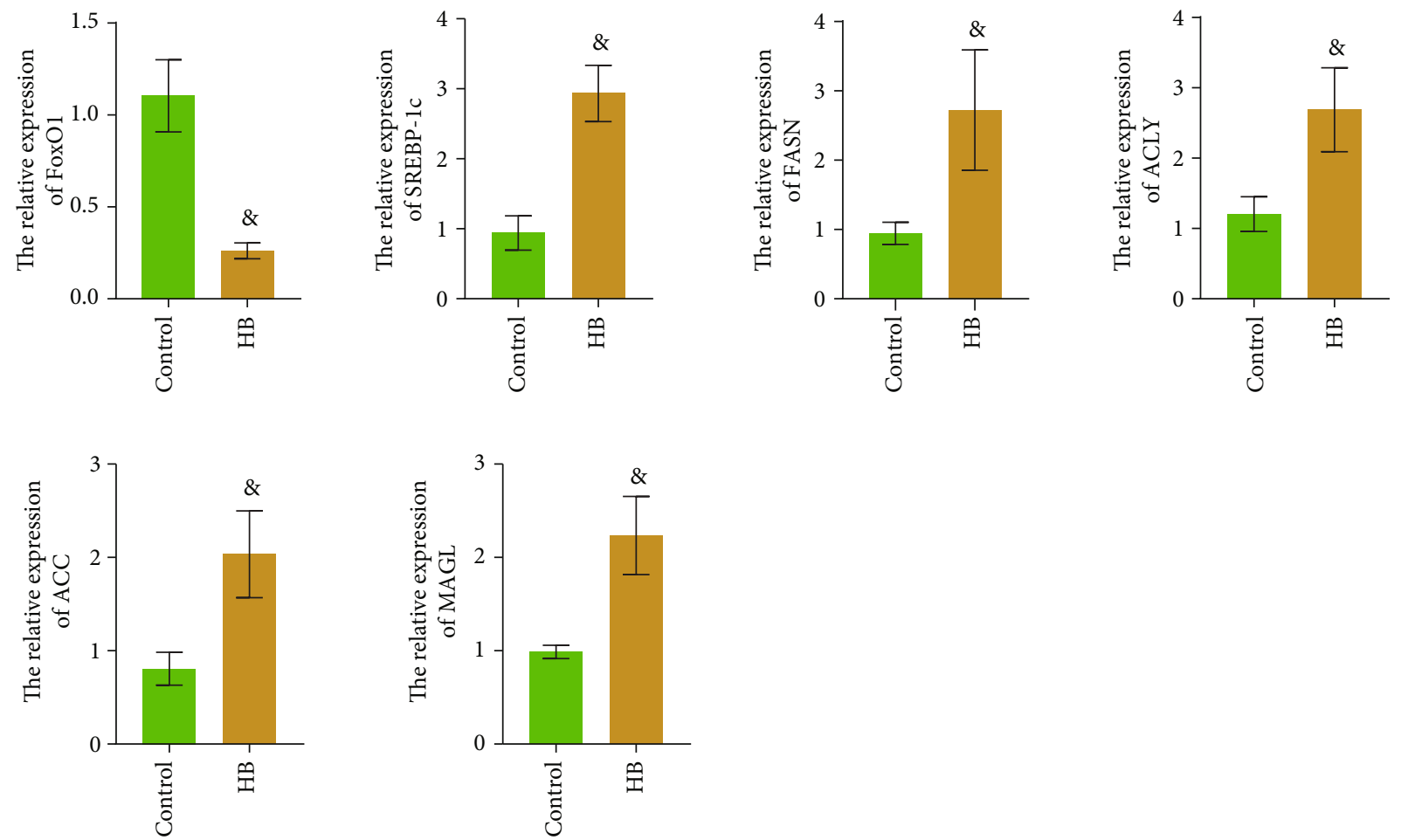

(a)
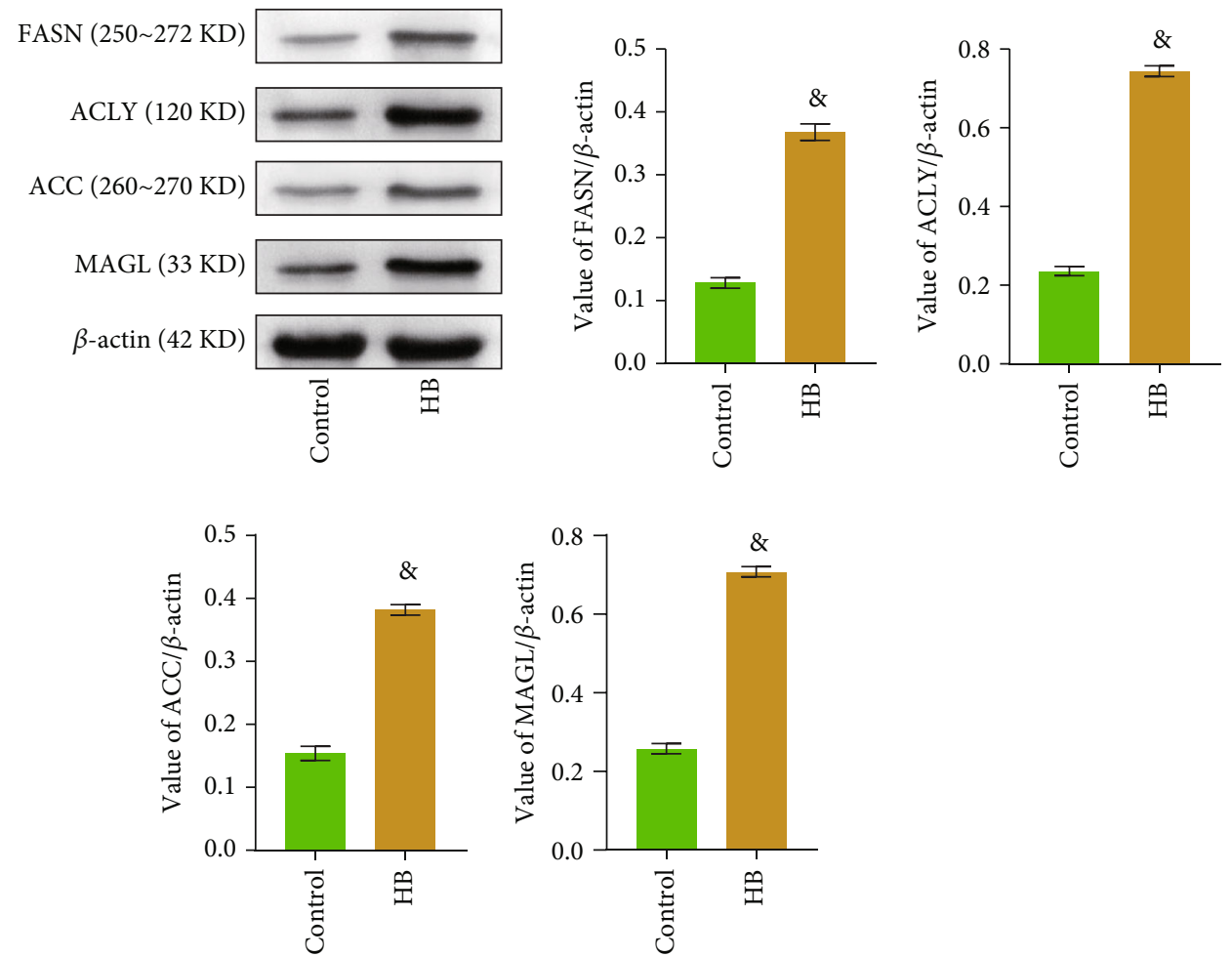

(b)

Figure 1: Continued. 


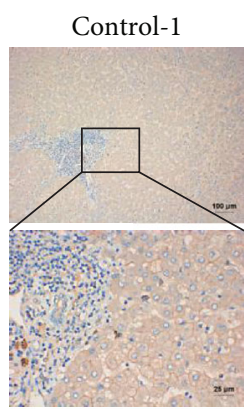

HB-1
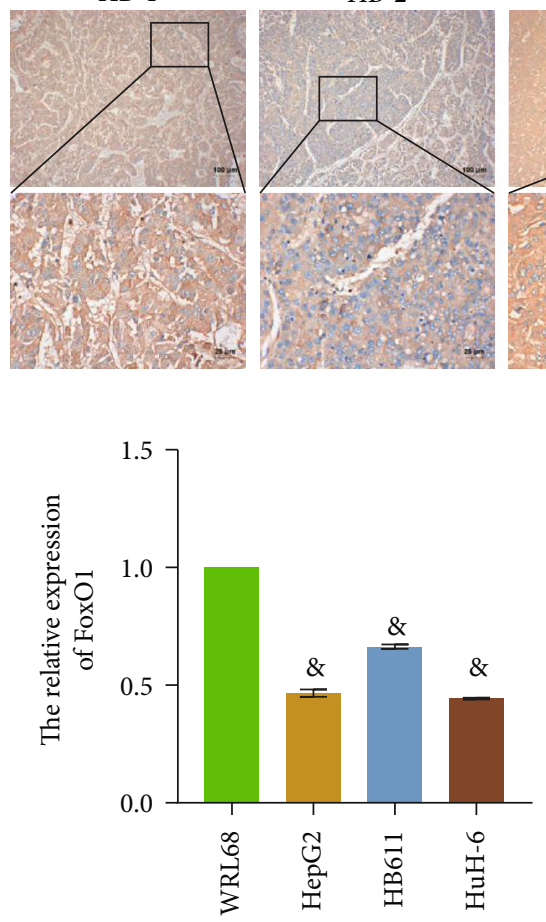

Control-3

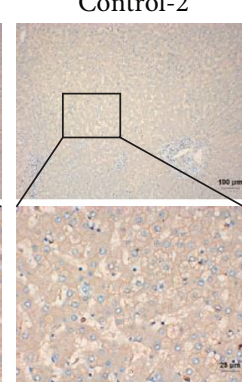

HB-2
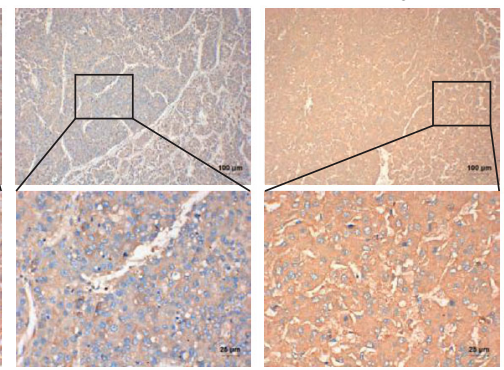

Control-4

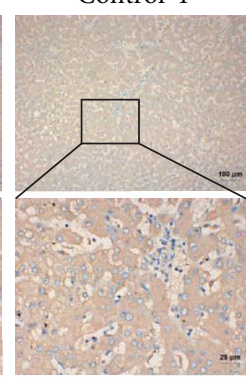

HB-4

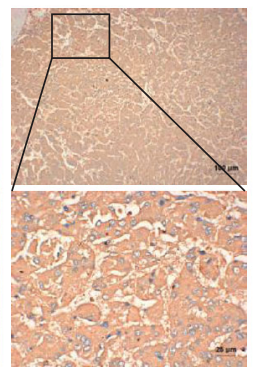

(c)

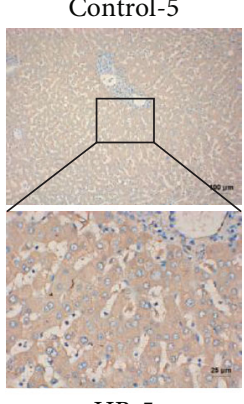

HB-5

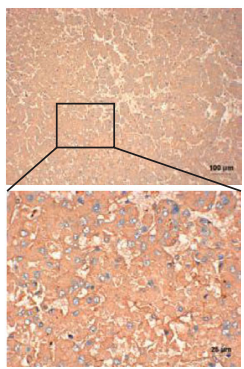

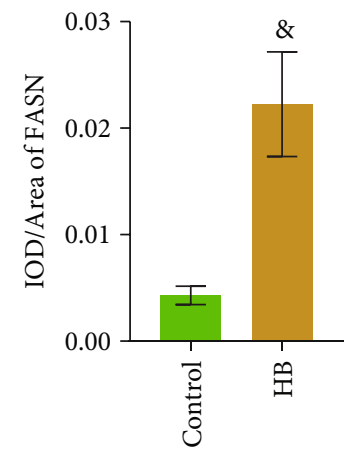
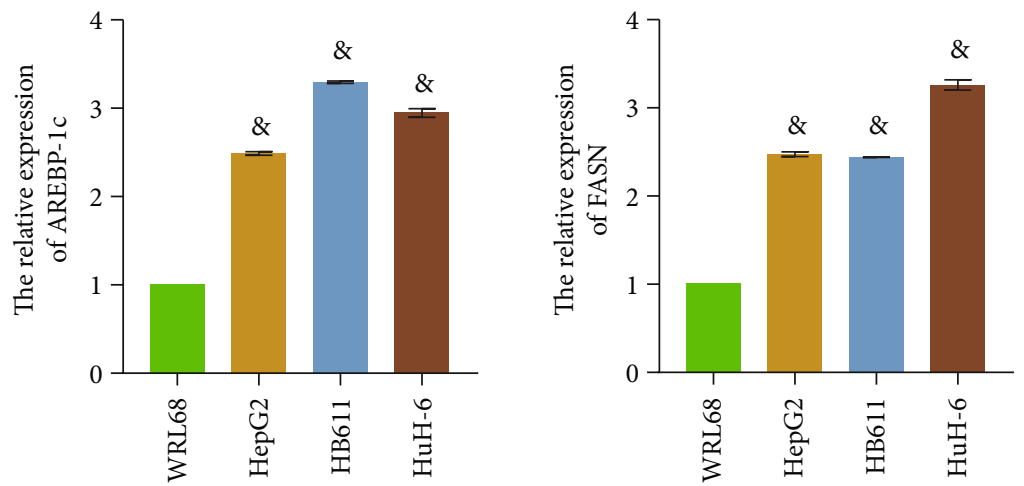

(d)

FIGURE 1: FoxO1 expression was downregulated while SREBP-1c and fatty acid metabolism genes were upregulated in HB. (a) The relative expression levels of FoxO1, SREBP-1c, FASN, ACLY, ACC, and MAGL were detected by RT-qPCR. (b) WB was used to measure the expressions of FASN, ACLY, ACC, and MAGL. (c) IHC evaluated the expression levels of FASN. (d) RT-qPCR was performed to examine the relative expressions of FoxO1, SREBP-1c, and FASN. The magnification is 100 or 400 times, and the corresponding scale bar is $100 \mu \mathrm{m}$ or $25 \mu \mathrm{m} ;{ }^{8} \mathrm{P}<0.05$ vs. the control group in $(\mathrm{a}-\mathrm{c}) ;{ }^{8} \mathrm{P}<0.05$ vs. the WRL68 group in (d). All experiments were performed 5 times.

scratched cells. Then, serum-free DMEM medium was added. After being cultured for $0 \mathrm{~h}, 24 \mathrm{~h}$, and $48 \mathrm{~h}$, the cells were photographed under an inverted biological microscope (DSZ2000X, Cnmicro).

2.8. Transwell Assay. Transwell chamber (\#3428, Corning) with a matrix gel (\#354262, BD) was used to perform the transwell assay. The cells were digested into single-cell suspension with trypsin and resuspended in serum-free medium to $2 \times 10^{6}$ cells $/ \mathrm{mL}$. $100 \mu \mathrm{L}$ cell suspension was inoculated in the up-compartment, and $500 \mu \mathrm{L} 10 \%$ DMEM/F12 medium (D8437, Sigma) was added in the low-compartment. It was incubated in an incubator at $37^{\circ} \mathrm{C}$ for $48 \mathrm{~h}$. The cells in the up-compartment were rinsed with PBS solution. Cells were fixed with paraformaldehyde for $20 \mathrm{~min}$, and the membrane was removed. The membrane was stained with $0.1 \%$ crystal violet for $5 \mathrm{~min}$. Cells on the outer surface of the upper compartment were observed under an inverted biological microscope. After decolorization by acetic acid immersion, the cells' absorbance at $550 \mathrm{~nm}$ was measured with a microplate analyzer [20].

2.9. Fatty Acid Metabolism Detection. Nanjing Jiancheng Bioengineering Institute produced the ATP Assay Kit (\#A095-1-1), Nonesterified Free Fatty Acids Assay Kit (\#A042-2-1), and Triglyceride (TG) Assay Kit (\#A110-1-1). Human Fatty Acid Oxidase (FAO) ELISA Kit (\#JL48747) and Human Acetyl-Coenzyme A (Acetyl-CoA) ELISA Kit (\#JL32777) were purchased from Jianglaibio (Shanghai, China). These kits were used to test adenosine triphosphate 

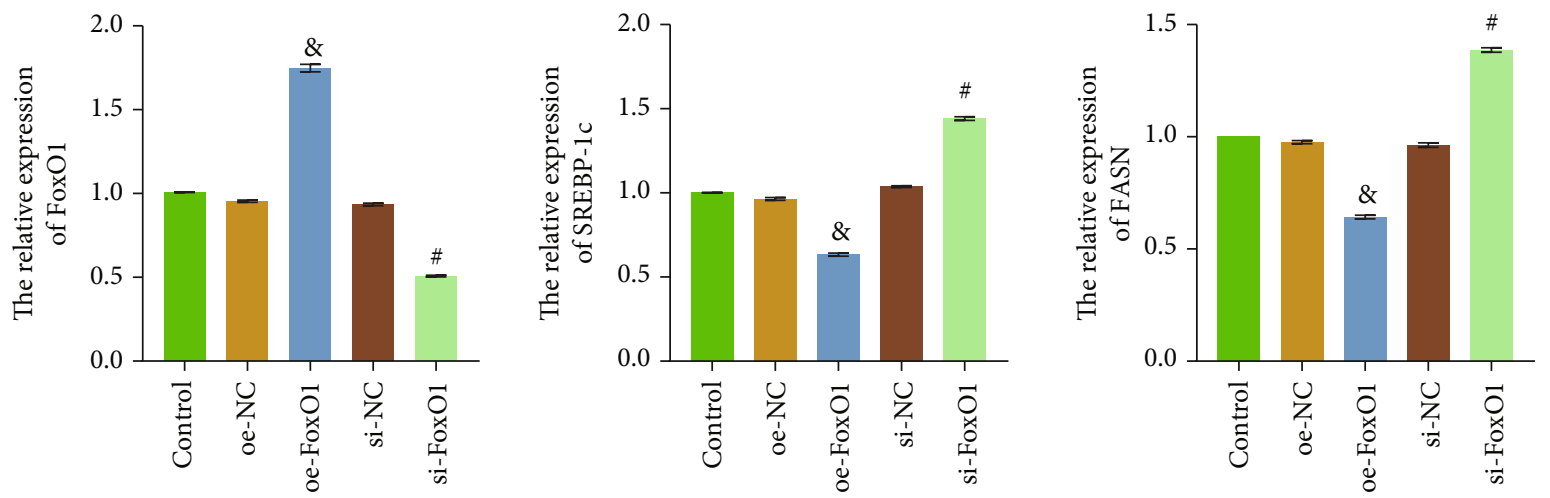

(a)

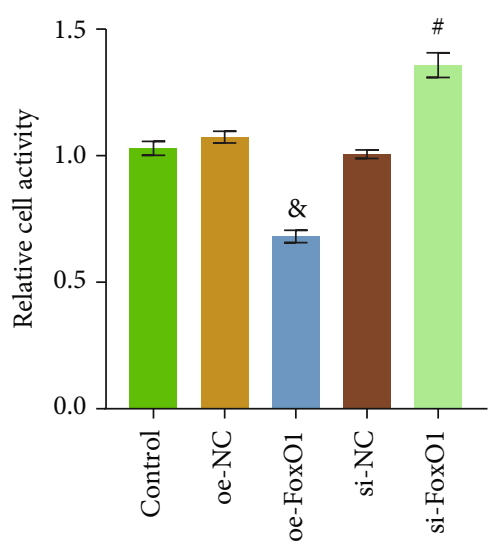

(b)

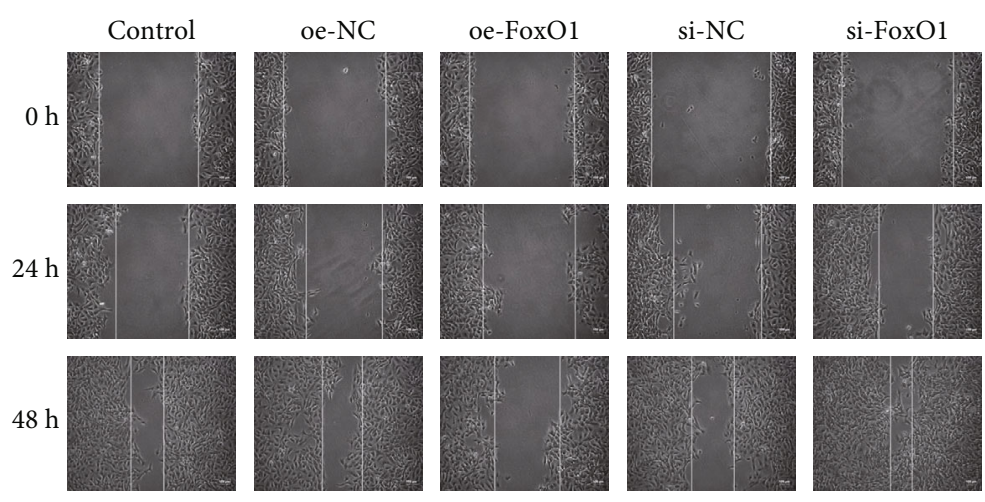

(c)

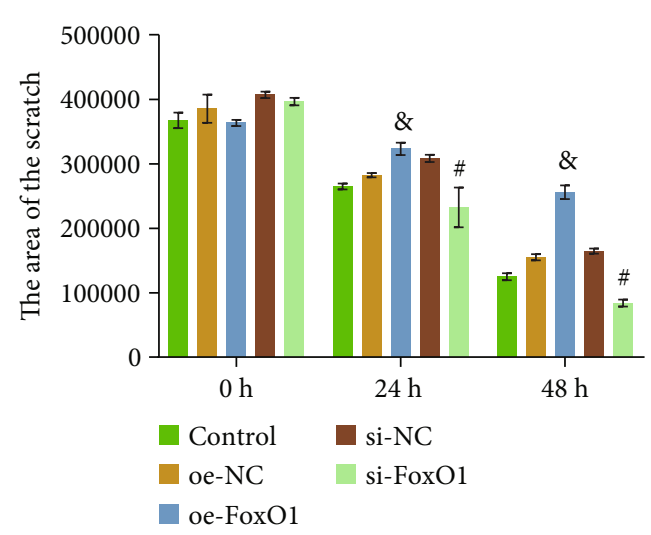

oe-FoxO1

FIgure 2: Continued. 

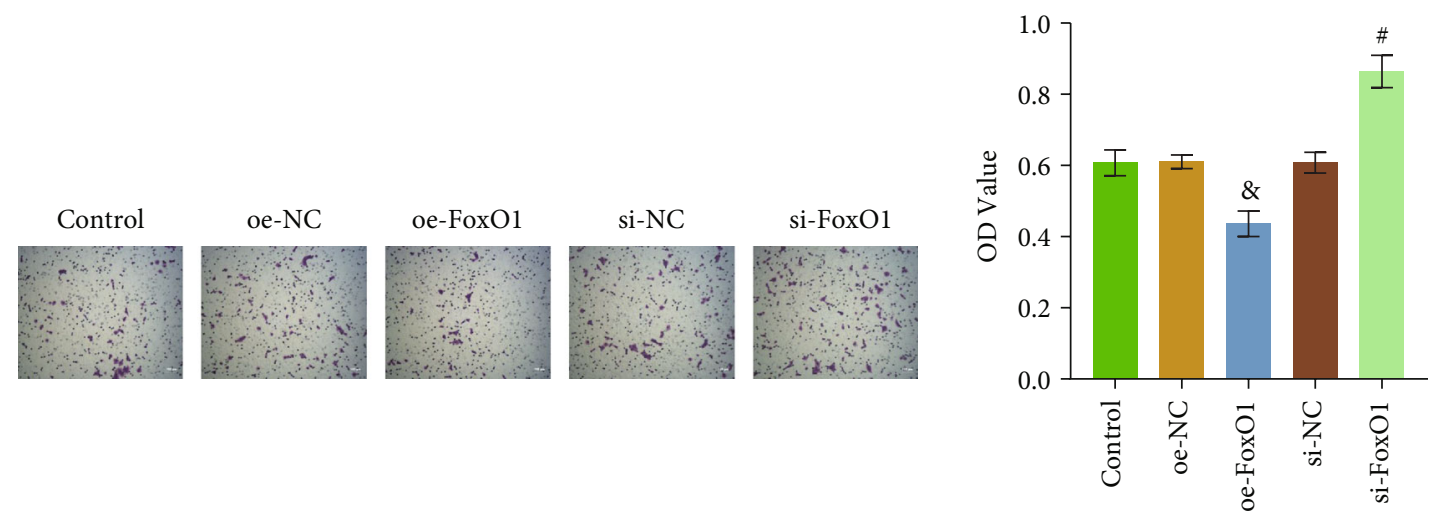

(d)

FIgURE 2: FoxO1 deficiency led to upregulation of SREBP-1c expression and enhanced proliferation, migration, invasion of HB. (a) RTqPCR detected the relative expression levels of FoxO1, SREBP-1c, and FASN. (b) CCK8 assay was used to detect cell proliferation. (c) Cell migration was evaluated by scratch assay. (d) Transwell assay was performed to examine cell invasion. The magnification is 100 times, scale bar $=100 \mu \mathrm{m} ;{ }^{\&} \mathrm{P}<0.05$ vs. the oe-NC group; ${ }^{\#} \mathrm{P}<0.05$ vs. the si-NC group. All experiments were performed 3 times.

(ATP), FAO, TG, nonesterified fatty acid (NEFA), and Acetyl-CoA in cells. Each step strictly follows the instructions of the manual. Finally, the light signal at the specified wavelength was detected with a microplate analyzer [21].

2.10. Chromatin Immunoprecipitation (ChIP). ChIP Kit (ab500, Abcam) was used to detect the direct interaction between SREBP-1c and FoxO1. After the cells were digested with trypsin, the cell suspension was incubated with formaldehyde and glycine to cross-link the target protein and the corresponding genomic DNA. Buffer D and protease inhibitors were added to the cell suspension. The mixture was ultrasonically crushed for $60 \mathrm{~s}$ and centrifuged. Agarose gel electrophoresis was performed to analyze the DNA fragment size. Then, immunoprecipitation was performed using agarose beads according to the instructions. Finally, the agarose beads were suspended with DNA purifying slurry to unlock the cross-linking and purify the DNA. Six pairs of primers were designed according to the FoxO1 gene's promoter (Table 2). RTqPCR amplified DNA, and Fold Enrichment was calculated using the $2^{-\triangle \Delta C T}$ method.

2.11. In Vivo Tumorigenesis. Eight-week-old female nude mice $(\mathrm{BALB} / \mathrm{c}, \mathrm{nu} / \mathrm{nu})$ were purchased from the Animal Center of Central South University. Mice were randomly divided into 5 groups $(n=9)$ : the control, the si-NC, the si-FoxO1, the si-SREBP-1c, and the si-FoxO1+si-SREBP-1c groups. Then, they were kept in captivity free of pathogens and given food and water at will. HepG2 cells were digested with trypsin and resuspended in a sterile salt solution. An equal number of HepG2 cells $\left(2 \times 10^{5}\right)$ was subcutaneously injected into the lower abdomen of nude mice [21]. Tumor volume was measured weekly until the maximum volume was $1000 \mathrm{~mm}^{3}$. All mice were sacrificed with the manual cervical dislocation method. Tumor tissues were removed, measured, weighed, and further examined.
2.12. Hematoxylin and Eosin (HE) Staining. The tumor tissues of mice were made into paraffin sections. The sections were baked in the microwave oven at $60^{\circ} \mathrm{C}$ for $2 \mathrm{~h}$. The sections were then deparaffinized in xylene and placed in $100 \%$, $100 \%, 95 \%, 85 \%$, and $75 \%$ ethanol for $5 \mathrm{~min}$ at each stage. The sections were soaked in distilled water and stained with hematoxylin for $5 \mathrm{~min}$ and eosin solution for $3 \mathrm{~min}$. Then, the sections were dehydrated in gradient alcohol and soaked in xylene two times, each $10 \mathrm{~min}$. Finally, they were sealed with neutral gum (Sigma), and photos were taken with an ordinary light microscope (BA210T, Motic).

2.13. Immunofluorescence (IF). A microwave oven was used for baking the paraffin sections of mouse tumor tissues at $60^{\circ} \mathrm{C}$ for $2 \mathrm{~h}$. The sections were deparaffinized and rehydrated by xylene and multiple concentration ethanol solutions. The slices were immersed in $\mathrm{pH} 6.0$ citrate buffer (Wellbio). The citrate buffer was heated by the microwave oven for $24 \mathrm{~min}$. After the buffer liquid was cooled, the sections were immersed in sodium borohydride solution for $30 \mathrm{~min}$ and Sudan Black solution for $5 \mathrm{~min}$. Sections were sealed with $10 \%$ normal serum for $60 \mathrm{~min}$. Sections were incubated overnight with primary antibody FASN (1:50, 10624-1-AP, Proteintech) at $4^{\circ} \mathrm{C}$. On the second day, sections were incubated with the secondary antibody at $37^{\circ} \mathrm{C}$ for $90 \mathrm{~min}$. Finally, sections were incubated with DAPI solution (Wellbio) at $37^{\circ} \mathrm{C}$ for $10 \mathrm{~min}$ and rinsed with PBS buffer. Sections were sealed with buffered glycerin and observed under a fluorescence microscope.

2.14. Statistical Analysis. Statistical analysis was performed using SPSS 20.0 (SPSS Inc, USA). Data were presented in the form of mean \pm standard deviation $(\mathrm{X} \pm \mathrm{SD})$. All experiments were repeated at least three times. The Student $t$-test was used to analyze the differences between the two groups. Comparisons among multiple groups were conducted by one-way analysis of variance, followed by Tukey's post hoc test. $P<0.05$ was considered statistically significant. 


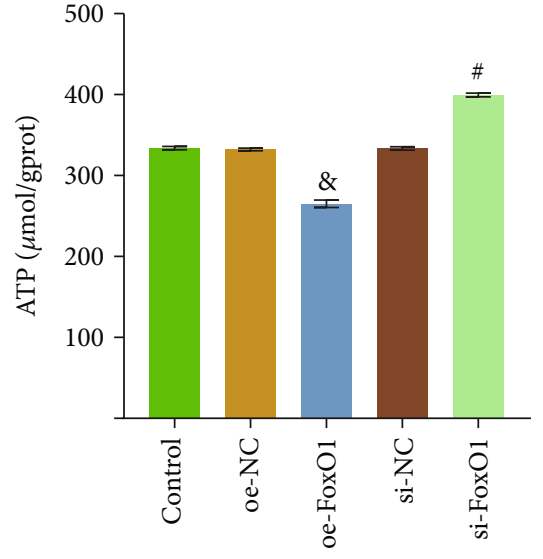

(a)

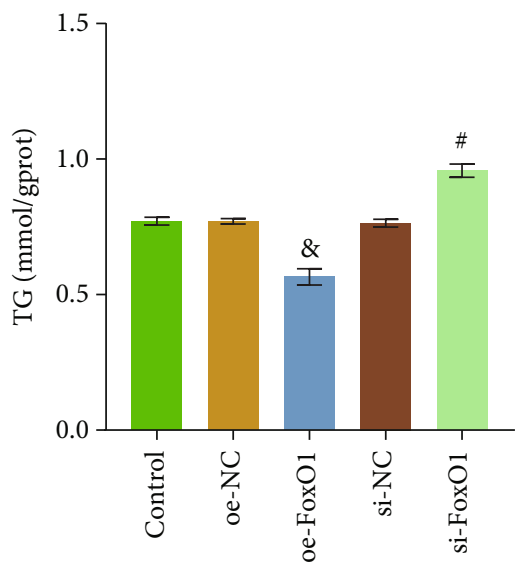

(c)

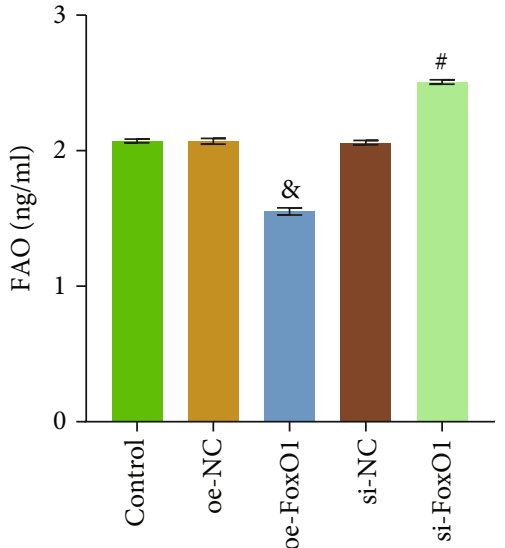

(b)

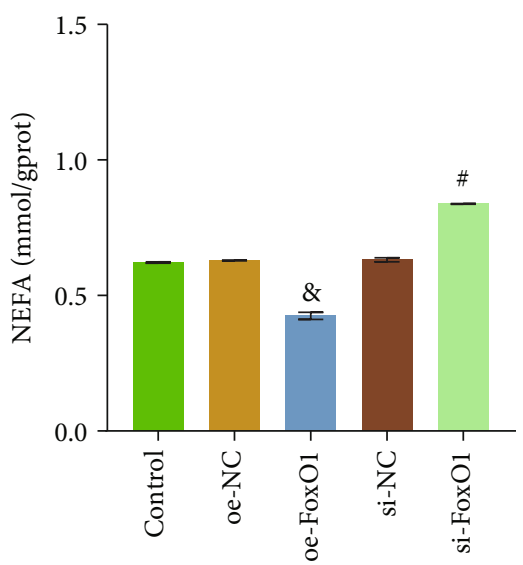

(d)

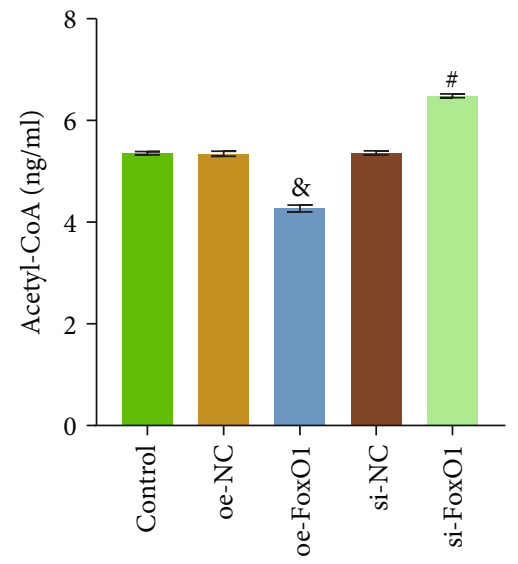

(e)

FIGURE 3: FoxO1 deficiency enhanced fatty acid metabolism in HB. (a) ATP Assay Kit was used to detect the concentration of total cellular ATP. (b) Human FAO ELISA Kit was adopted to examine FAO. (c) TG Assay Kit detected TG. (d) NEFA was examined with Nonesterified Free Fatty Acids Assay Kit. (e) Cellular Acetyl-CoA level was evaluated with Human Acetyl-CoA ELISA Kit. ${ }^{\circledR} P<0.05$ vs. the oe-NC group; ${ }^{\#} P<0.05$ vs. the si-NC group. All experiments were performed 3 times.

\section{Results}

3.1. FoxO1 Expression Was Downregulated while SREBP-1c and Fatty Acid Metabolism Genes Were Upregulated in $H B$. In order to explore the changes of fatty acid metabolism in $\mathrm{HB}, \mathrm{RT}-\mathrm{qPCR}$ and WB detected the expressions of FoxO1,
SREBP-1c, FASN, ACLY, ACC, and MAGL in clinical samples. As shown from Figure 1(a), compared with the control group, the FoxO1 expression in the $\mathrm{HB}$ group was decreased, and the SREBP-1c expression was significantly increased. At the level of RNA and protein, the expression levels of fatty acid metabolism-related indexes (FASN, ACLY, ACC, and 


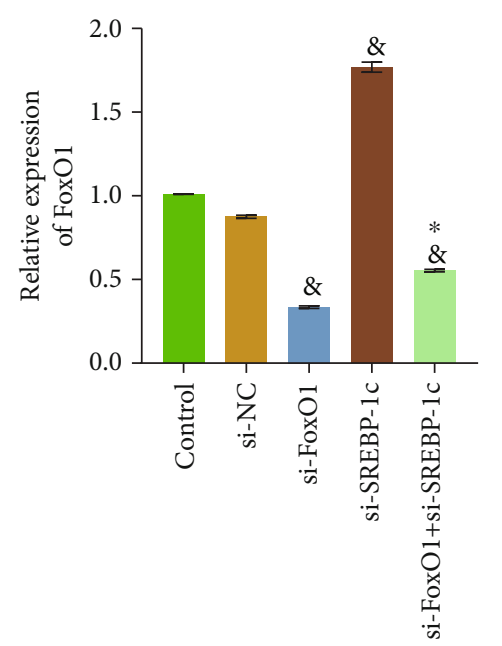

(a)

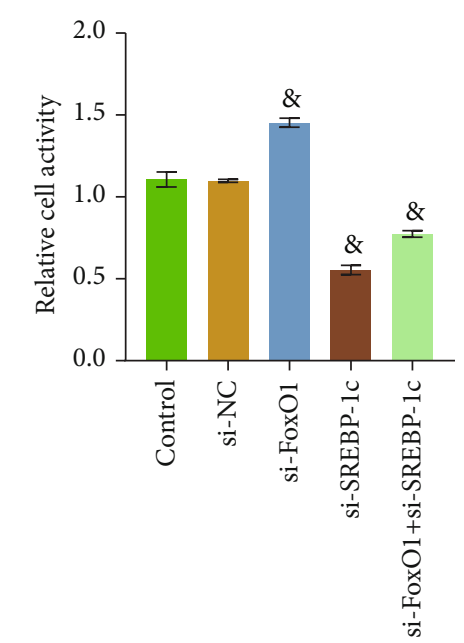

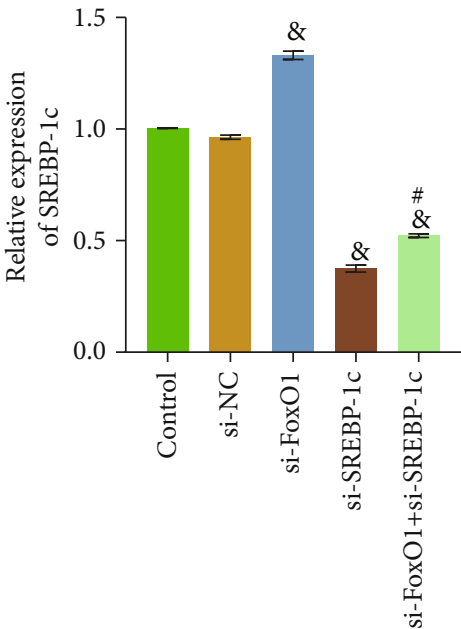

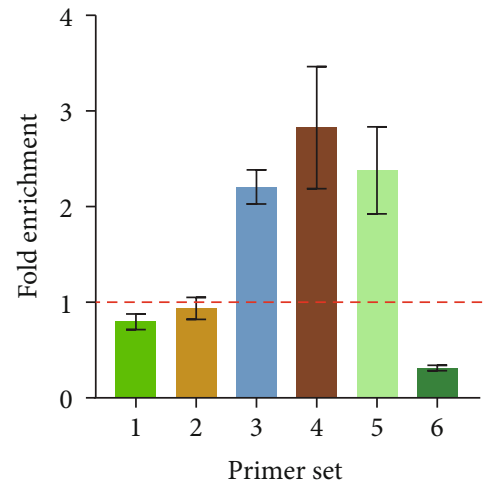

(b)

(c)

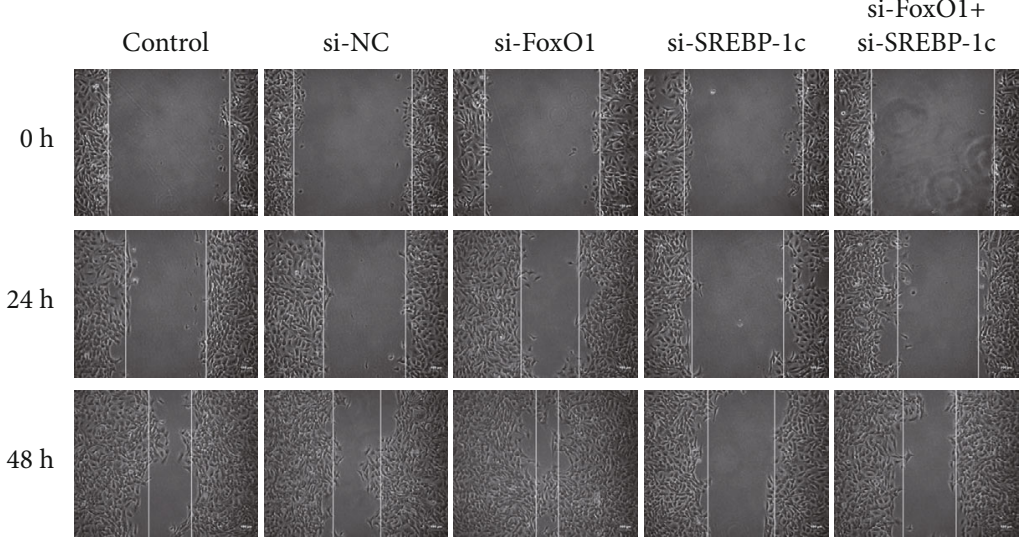

(d)

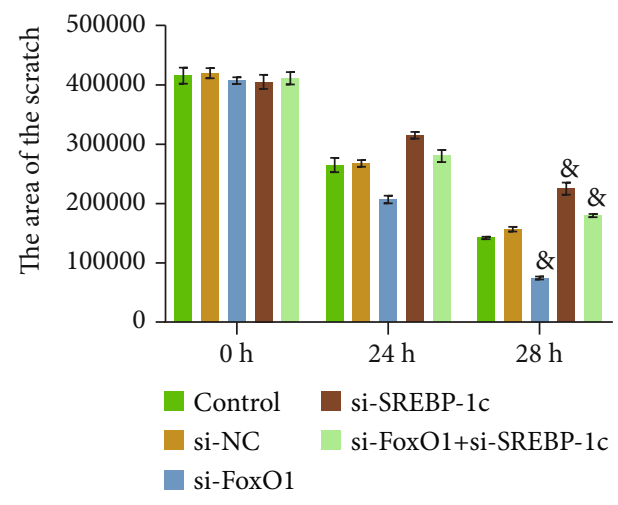

FIgURE 4: Continued. 

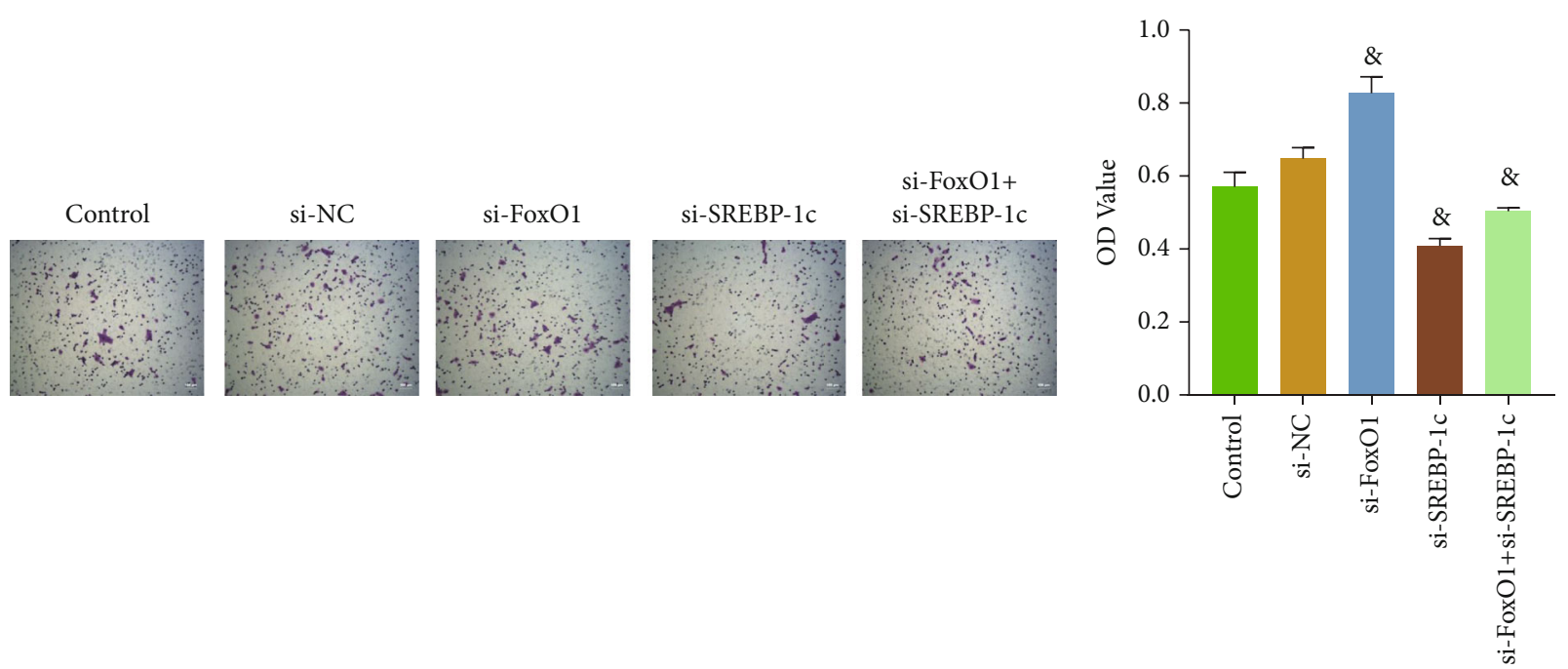

(e)

FIGURE 4: FoxO1 and SREBP-1c inhibited each other and regulated Huh-6 cell functions. (a) RT-qPCR detected the relative expression levels of FoxO1 and SREBP-1c. (b) ChIP was performed to detect the direct interaction between the FoxO1 gene and SREBP-1c protein. (c) CCK8 assay was used to detect cell proliferation. (d) Cell migration was evaluated by scratch assay. (e) Transwell assay was performed to examine cell invasion. The magnification is 100 times, scale bar $=100 \mu \mathrm{m} ;{ }^{\circledR} P<0.05$ vs. the si-NC group, ${ }^{*} P<0.05$ vs. the si-FoxO1 group, and ${ }^{\#} P<0.05$ vs. the si-SREBP-1c group. All experiments were performed 3 times.

MAGL) in the HB group were higher than those in the control group (Figures 1(a) and 1(b)). IHC results showed that FASN expression was upregulated in the HB group compared with the control group (Figure 1(c)). As shown in Figure $1(\mathrm{~d})$, among the three kinds of $\mathrm{HB}$, the difference in gene expression between Huh-6 cells and normal liver cells (WRL68) was the most significant, so Huh-6 cells were selected for subsequent experiments.

3.2. FoxO1 Deficiency Led to Upregulation of SREBP-1c Expression and Enhanced Proliferation, Migration, Invasion of $\mathrm{HB}$. In order to determine the effect of FoxO1 on SREBP-1 expression and cell function in HB, Huh-6 cells with FoxO1 overexpression or knockdown were constructed. RT-qPCR detected the expression levels of FoxO1, SREBP$1 \mathrm{c}$, and FASN, and the results suggested that FoxO1 overexpression or knockdown cells were completed (Figure 2(a)). Compared with the oe-NC group, the expression of SREBP-1c and FASN in the oe-FoxO1 group decreased. Compared with the si-NC group, the expression levels of SREBP-1c and FASN in the si-FoxO1 group were increased (Figure 2(a)). As shown in Figures 2(b)-2(d), cell proliferation, migration, and invasion abilities in the oe-FoxO1 group were weaker than those in the oe-NC group. Compared with the si-NC group, cell proliferation, migration, and invasion abilities of the si-FoxO1 group were enhanced. In other words, FoxO1 could inhibit the expression of SREBP-1c and FASN and reduce the proliferation, migration, and invasion abilities of Huh- 6 cells.

3.3. FoxO1 Deficiency Enhanced Fatty Acid Metabolism in $H B$. Intracellular fatty acid metabolism in both overexpression and deletion of FoxO1 was examined to clarify the effect of FoxO1 on fat metabolism in HB. As shown in
Figures 3(a)-3(e), compared with the oe-NC group, ATP, FAO, TG, NEFA, and Acetyl-CoA contents in the oeFoxO1 group decreased. In the meantime, compared with the si-NC group, the contents of ATP, FAO, TG, NEFA, and Acetyl-CoA in the si-FoxO1 group increased (Figures 3(a)-3(e)). These results suggested that FoxO1 could inhibit the uptake and production of ATP, TG, NEFA, and Acetyl-CoA. It also inhibits the FAO. In other words, FoxO1 inhibited fatty acid metabolism (including anabolism and catabolism) in Huh- 6 cells.

3.4. FoxO1 and SREBP-1c Inhibited Each Other and Regulated Huh-6 Cell Functions. It has been previously confirmed that FoxO1 has a regulatory effect on SREBP-1c and HB. To further explore how FoxO1 and SREBP-1c work together, Huh- 6 cells that FoxO1 and SREBP-1c knockdown at the same time or SREBP-1c knockdown alone were constructed. Figure 4(a) showed that SREBP-1c could inhibit FoxO1 expression (fold change $\approx 1.65$ ), and FoxO1 also inhibited SREBP-1c expression (fold change $\approx 1.39$ ). We could see that SREBP-1c had a more substantial inhibitory effect on FoxO1 than FoxO1 on SREBP-1c (fold change $\approx 1.65>1.39$ ). Then, ChIP was performed to study whether there was a direct interaction between SREBP-1c and FoxO1 gene. As shown in Figure 4(b), the Fold Enrichment of primers 3, 4, and 5 was greater than 1, indicating that the enrichment capacity of nonspecific adsorption of antibodies was less than the specific action of antibodies. It showed that SREBP-1c directly interacted with sites 3, 4, and 5 of the FoxO1 gene's promoter. Cell function results exhibited that compared with the si-NC group, the cells' proliferation, migration, and invasion abilities in the si-SREBP-1c group were reduced. In contrast, those in the si-FoxO1 group were enhanced (Figures $4(\mathrm{c})-4(\mathrm{e})$ ). When 


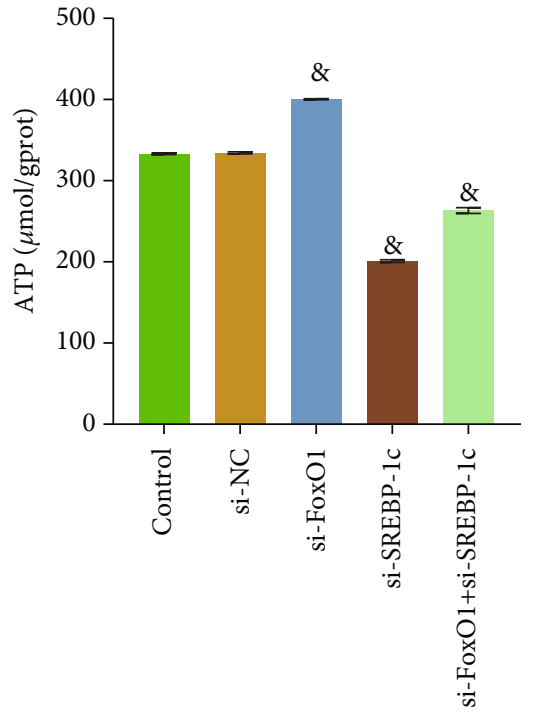

(a)

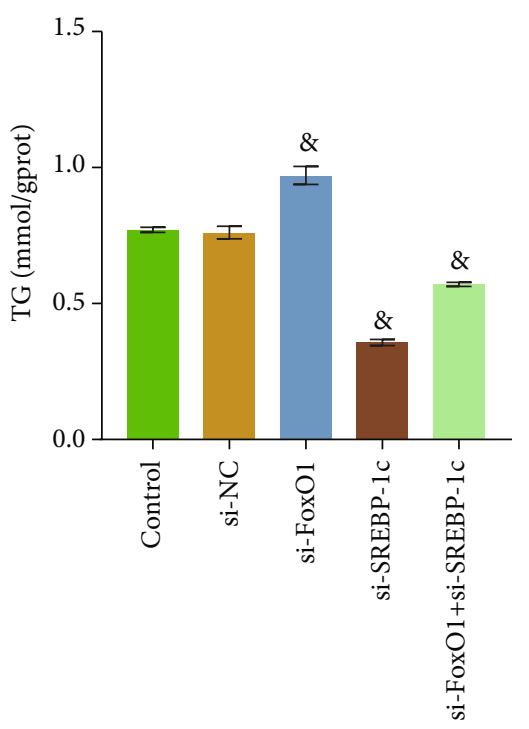

(c)

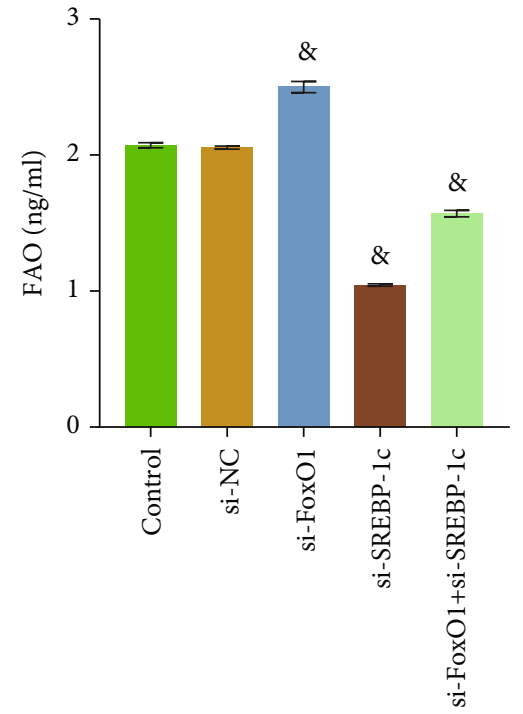

(b)

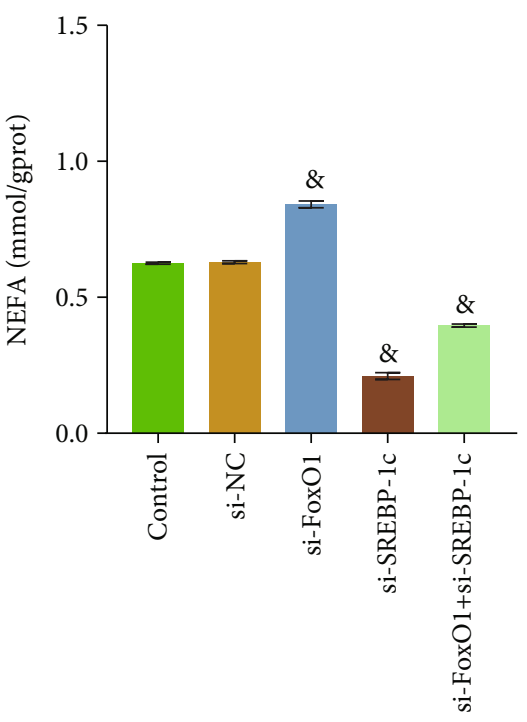

(d)

FIgURe 5: Continued. 


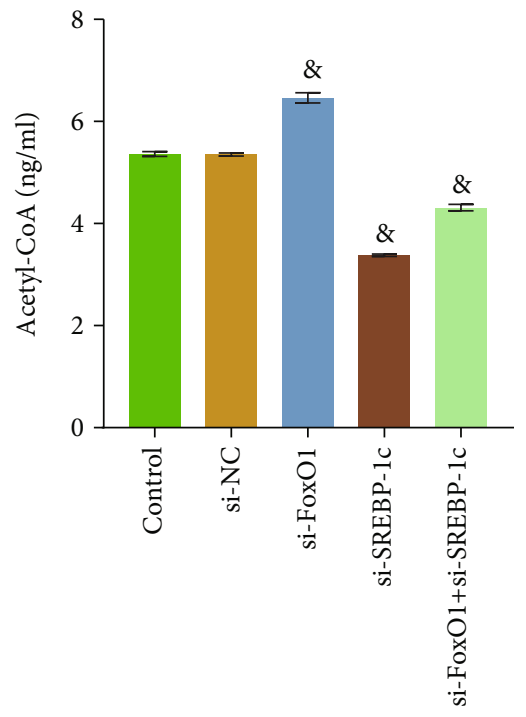

(e)

FIgure 5: Coordinated regulation of FoxO1 and SREBP-1c regulated fatty acid in Huh-6 cells. (a) ATP Assay Kit was used to detect the concentration of total cellular ATP. (b) Human FAO ELISA Kit was adopted to examine FAO. (c) TG Assay Kit detected TG. (d) NEFA was examined with Nonesterified Free Fatty Acids Assay Kit. (e) Cellular Acetyl-CoA level was evaluated with Human Acetyl-CoA ELISA Kit. ${ }^{\circledR} P<0.05$ vs. the si-NC group. All experiments were performed 3 times.

FoxO1 and SREBP-1c were knocked down simultaneously, cells' proliferation, migration, and invasion abilities were reduced. These results suggested that FoxO1 inhibited cell proliferation, migration, and invasion, while SREBP-1c had the opposite effect. Meanwhile, FoxO1 and SREBP-1c inhibit each other, and their net effect in Huh-6 cells was to promote cell proliferation, migration, and invasion.

3.5. Coordinated Regulation of FoxO1 and SREBP-1c Regulated Fatty Acid Metabolism in Huh-6 Cells. We have demonstrated that FoxO1 has a regulatory effect on SREBP-1c and fatty acid metabolism in HB cells. To study how FoxO1 and SREBP-1c play roles in regulating fatty acid metabolism, we constructed Huh- 6 cells that knocked down both FoxO1 and SREBP-1c or knocked down SREBP-1c alone for the detection of fatty acid metabolism-related indicators. As shown in Figures 5(a)-5(e), compared with the siNC group, the contents of ATP, FAO, TG, NEFA, and Acetyl-CoA decreased in the si-FoxO1+si-SREBP-1c group and the si-SREBP-1c group, while that increased in the siFoxO1 group. These results indicated that the net effect of FoxO1 and SREBP-1c was to promote fatty acid metabolism in $\mathrm{HB}$ cells.

3.6. Coordinated Regulation of FoxO1 and SREBP-1c Facilitated the Progression of $H B$ by Regulating Fatty Acid Metabolism In Vivo. Previous experiments were all conducted in vitro. Subcutaneous tumor-forming models of nude mice were constructed, and tumor tissues were collected to verify whether the results of the in vivo experiments were consistent with those in vitro. Figure 6(a) showed that the subcutaneous tumorigenesis model of nude mice was successfully constructed. As shown from Figure 6(b), com- pared with the si-NC group, the tumor volume and weight of the si-FoxO1 group increased significantly, while those of the si-SREBP-1c group decreased. RT-qPCR results demonstrated that knockdown of FoxO1 and SREBP-1c genes was successfully realized in tumor tissues (Figure 6(c)). As shown in Figure 6(d), compared with the si-NC group, the tumor tissue structure and morphology were regular and tightly arranged in the si-FoxO1 group. In si-SREBP-1c and si-FoxO1+si-SREBP-1c groups, the structure of tumor tissues was damaged. Compared with the si-NC group, the expression levels of FASN, ACLY, ACC, and MAGL in the si-FoxO1 group were increased in the si-SREBP-1c and siFoxO1+si-SREBP-1c groups were decreased (Figures 7(a)$7(c)$ ). Tumor tissue detection results showed that FoxO1 and SREBP-1c inhibited each other, and the net effect of FoxO1 and SREBP-1c facilitated the progression of HB by regulating fatty acid metabolism in vivo.

\section{Discussion}

Metabolic reprogramming in cancer cells has been recognized as one of the basic features of cancer [22]. In gastric cancer, SREBP-1c is activated and fatty acid synthesis is significantly increased [23]. Neoadipogenesis and fatty acid $\beta$ oxidation are very active in hepatocellular carcinoma [24]. Our analysis of clinical samples indicates that the expression of FoxO1 was downregulated in $\mathrm{HB}$ tissues, and the expression of genes of SREBP-1c and key enzymes in fatty acid metabolism were significantly upregulated. Naturally, the activation of SREBP-1c and fatty acid metabolism is preliminarily considered a significant characteristic of HB. FoxO1 degradation promotes cell proliferation in colon cancer [25]. Interestingly, FoxO1 overexpression in esophageal 


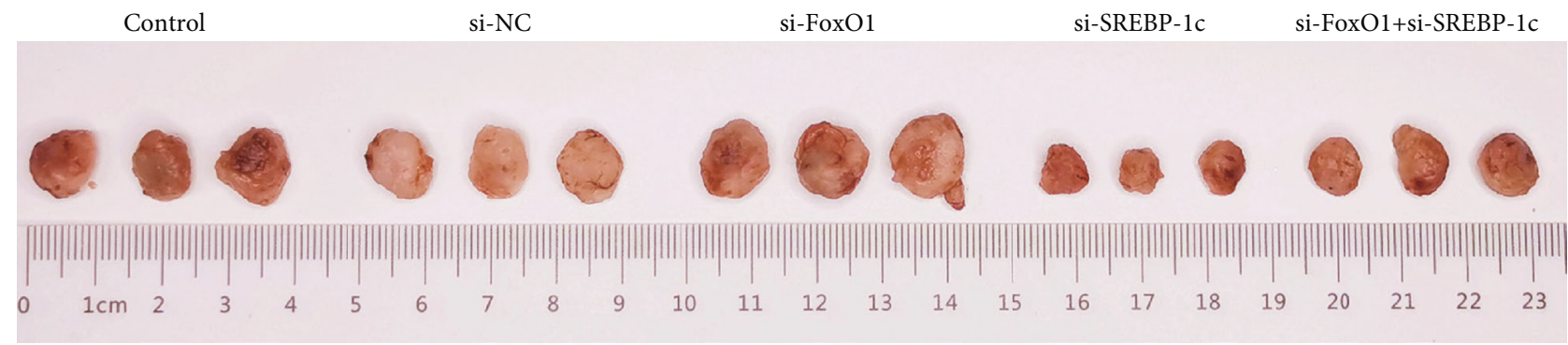

(a)
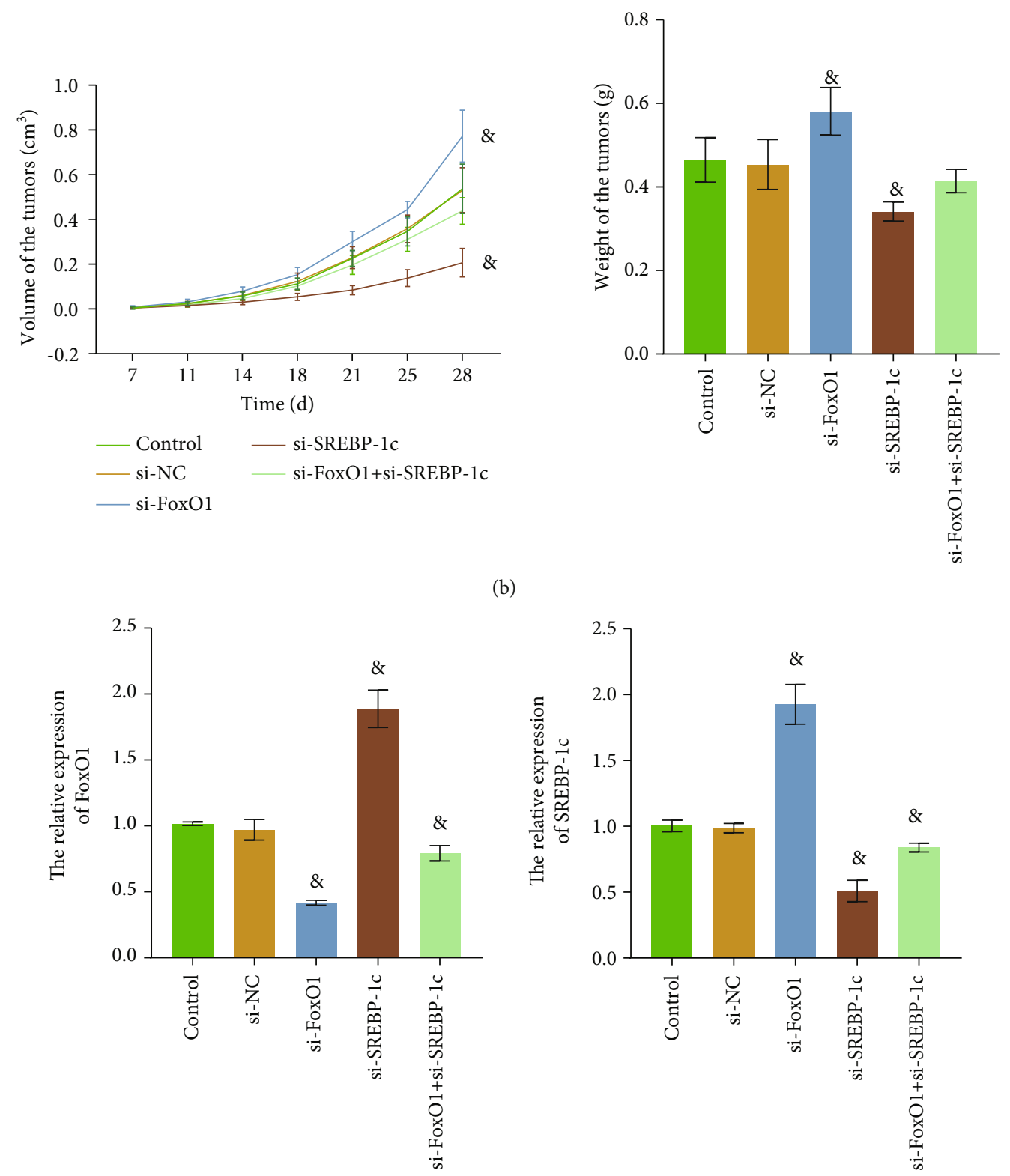

(b)

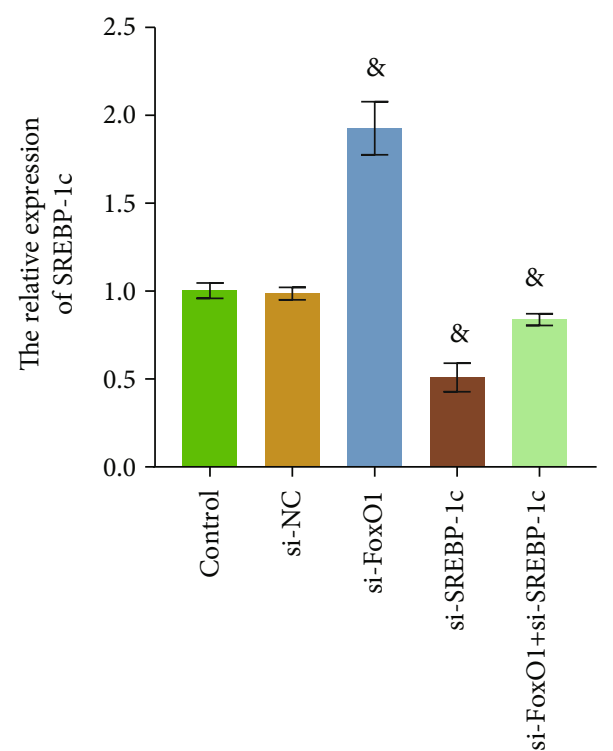

(c)

Figure 6: Continued. 

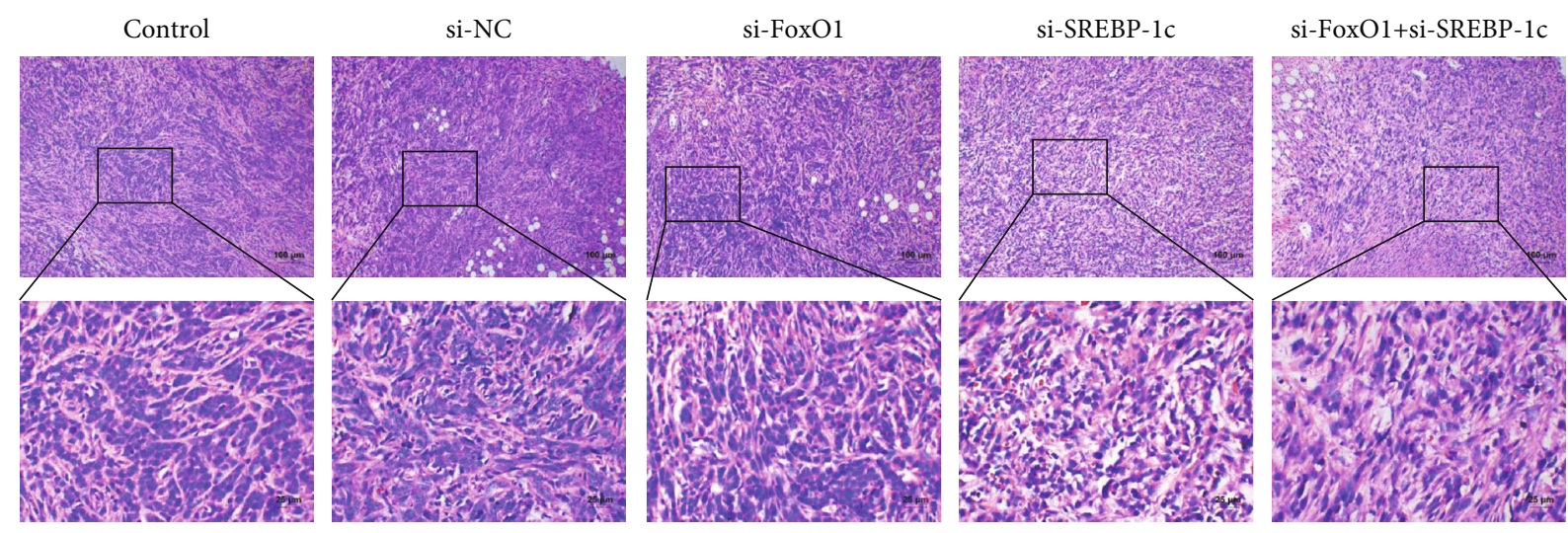

(d)

FIgURE 6: Coordinated regulation of FoxO1 and SREBP-1c facilitated the progression of HB in vivo. (a) Photograph of subcutaneous neoplasia in nude mice. (b) Volume and weight of tumor tissues. (c) The relative expression levels of FoxO1 and SREBP-1c were detected by RT-qPCR. (d) HE staining was performed to observe tumor tissues. The magnification is 100 or 400 times, and the corresponding scale bar is $100 \mu \mathrm{m}$ or $25 \mu \mathrm{m}$; ${ }^{\circledR} P<0.05$ vs. the si-NC group. All experiments were performed 3 times.

cancer promotes tumor development by increasing macrophage infiltration [26]. After cervical cancer, the proliferation, migration, and invasion abilities are significantly enhanced [27]. In summary, FoxO1 has various functions and regulates the progression of multiple types of cancer through numerous pathways. In this study, we find that the knockdown of FoxO1 promotes the development of HB. SREBP-1c is a crucial protein in fatty acid metabolism [28]. It activates the transcription of FASN, a major fatgenerating gene, which promotes the growth of bladder cancer [29]. In our study, when SREBP-1c was knocked down, the proliferation, migration, invasion, and division abilities of $\mathrm{HB}$ cells were reduced, and the fatty acid metabolism level was also significantly decreased.

In the study of diabetic cardiomyopathy, Ying et al. found that FoxO1 has a regulatory effect on fatty acid metabolism [30]. The ATP level of cancer cells is much higher than that of normal differentiated cells to meet the energy needs of growth and proliferation [31]. Usually, differentiated cells rely primarily on mitochondrial oxidative phosphorylation to produce ATP, a process that uses three main biofuels: glucose, glutamine, and fatty acids, while the proliferation of cancer cells depends on the FAO [32]. FAO is significantly enhanced in human glioblastoma, and inhibition of FAO leads to decreased intracellular ATP level and viability [33]. We detected ATP and $\mathrm{FAO}$ in $\mathrm{HB}$. Results indicated that the knockdown of FoxO1 could significantly increase FAO in $\mathrm{HB}$ and keep ATP at a high level. In other words, FoxO1 deficiency promotes catabolism of fatty acids in HB. Acetyl-CoA is a precursor of fatty acid and cholesterol synthesis [34]. ACLY-dependent Acetyl-CoA production plays a crucial role in the early stages of pancreatic neoplasia [35]. NEFAs are organic compounds with variable linear chain lengths of 6-32 carbons and hydrophilic heads containing a carboxylic acid, promoting colon, lung, skin, and breast cancer [36]. Lipids stored in lung neutrophils are trans- ported to metastatic tumor cells through the micropinocytosis-lysosome pathway, which enhances the survival and proliferation of tumor cells [37]. Our results showed that FoxO1 knockdown significantly increased Acetyl-CoA, NEFA, and TG levels in HB. In other words, FoxO1 deficiency can promote the anabolism of fatty acids in HB. Epigallocatechin gallate suppresses hepatic cholesterol synthesis by targeting SREBP-2 through SIRT1/FoxO1 signaling pathway [38]. In our study, FoxO1 knockdown accelerated HB cells' energy production, enhanced fatty acid metabolism, and ultimately promoted the development of $\mathrm{HB}$ cells. This result is consistent with previous studies.

Many studies have shown that FoxO1 could affect fatty acid metabolism by regulating the expression of SREBP-1c. For example, knockdown of FoxO1 significantly increased SREBP-1c and FASN in hepatitis C virus-infected cells [39]. Deng et al. found that FoxO1 could disrupt the assembly of key components of the SREBP-1c promoter transcription complex and inhibit the activity of the SREBP-1c promoter, thereby inhibiting the expression of SREBP-1c [40]. However, the mechanism by which FoxO1 plays a role in $\mathrm{HB}$ remained unclear. Through knockdown and overexpression of FoxO1, our study proved that FoxO1 could also inhibit the expression of SREBP-1c and inhibit fatty acid metabolism in HB. Interestingly, we found that SREBP-1c also had an inhibitory effect on FoxO1 (Figure 4(a)). There were few reports about the inhibitory effect of SREBP-1c on FoxO1, so we performed a ChIP experiment to prove the direct interaction between SREBP-1c and FoxO1. Some studies also supported that SREBP-1c could inhibit the expression of FoxO1 through indirect action. For example, Sajan et al. found that atypical protein kinase $\mathrm{C}$ activated by SREBP-1c inactivated FoxO1 via WD40/PROF (a scaffold protein)-associated Akt in diabetes [41]. Therefore, it was essential to determine their net effect on $\mathrm{HB}$ growth. We knocked down 

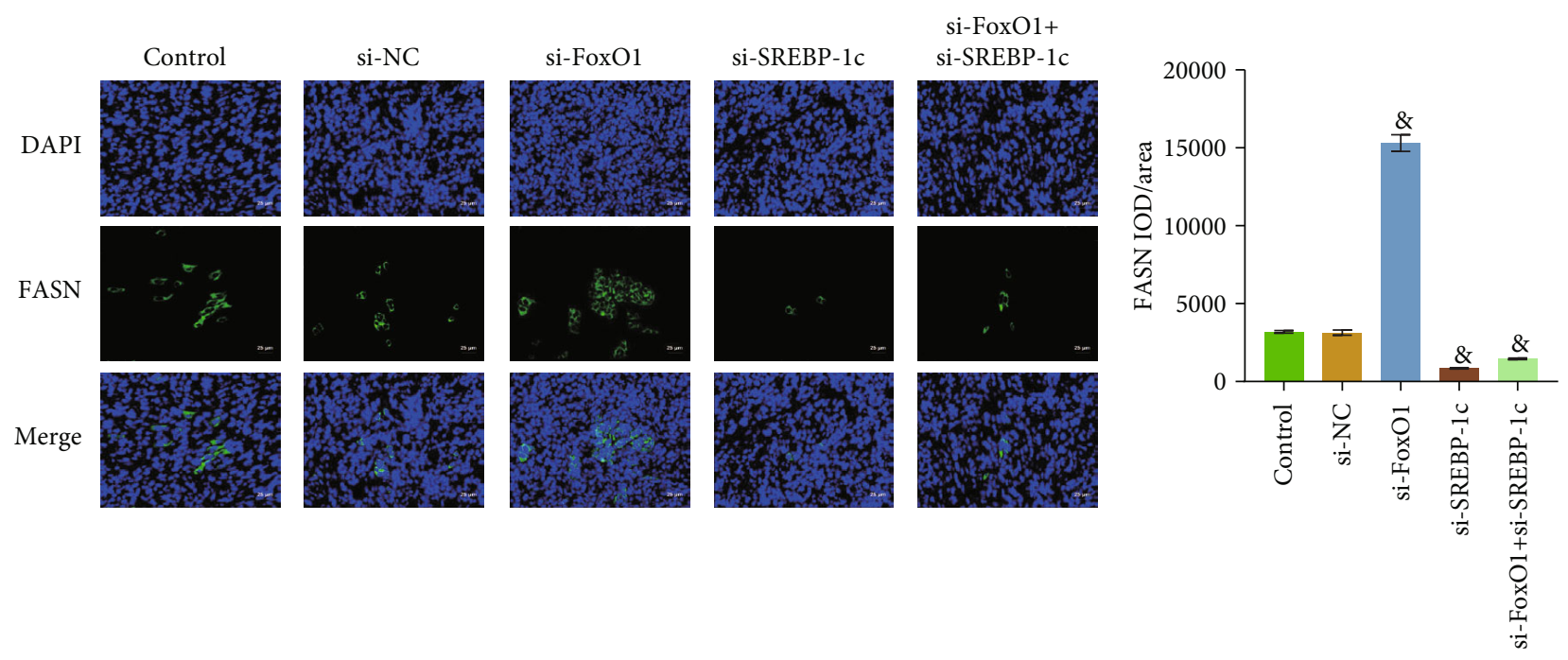

(a)
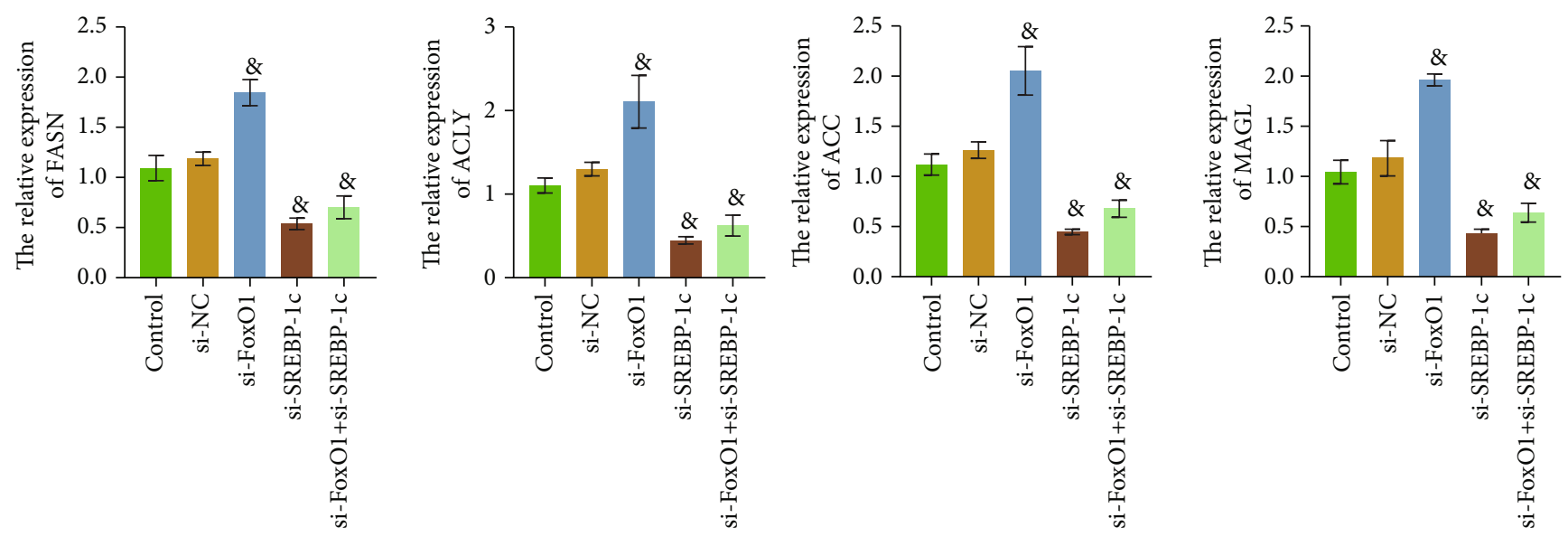

(b)

FIgURe 7: Continued. 

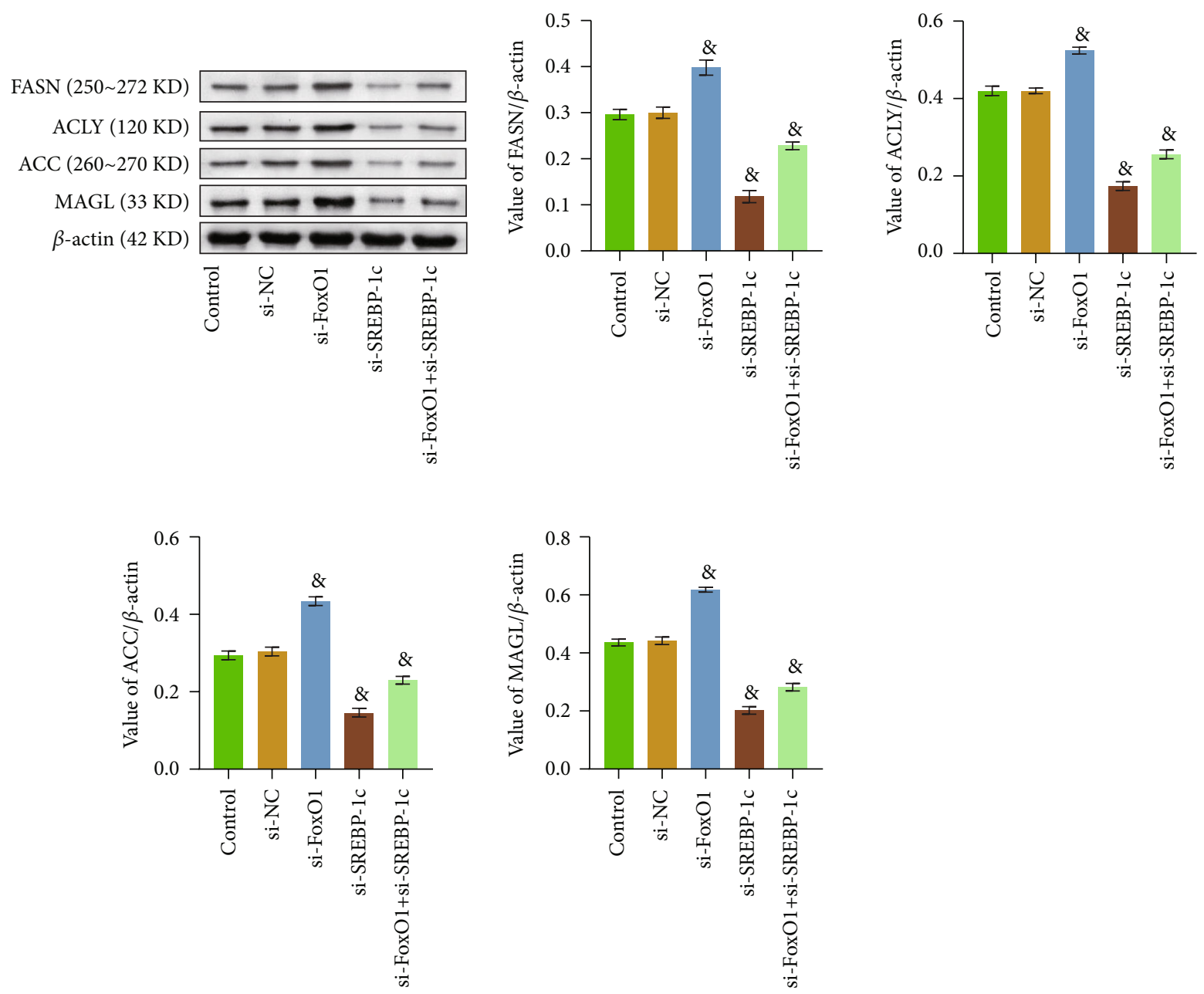

(c)

FIGURE 7: Coordinated regulation of FoxO1 and SREBP-1c regulated fatty acid metabolism in vivo. (a) The concentration of FASN was detected by IF. (b) The relative expression levels of FASN, ACLY, ACC, and MAGL were evaluated by RT-qPCR. (c) WB was used to measure the expressions of FASN, ACLY, ACC, and MAGL. The magnification is 400 times, scale bar $=25 \mu \mathrm{m}$; ${ }^{8} P<0.05$ vs. the si-NC group. All experiments were performed 3 times.

FoxO1 and SREBP-1c simultaneously in Huh-6 cells and found that fatty acid metabolism of Huh-6 cells was inhibited, and cell function and tumor-forming ability were weakened. In other words, coordinated regulation of FoxO1 and SREBP-1c could facilitate the progression of HB by regulating fatty acid metabolism.

In this study, we found mutual inhibition of FoxO1 and SREBP-1c in HB. In addition, SREBP-1c could bind to the promoter of FoxO1 to regulate its transcription. However, the mechanism of how FoxO1 inhibits SREBP$1 c$ expression remains unclear. As mentioned earlier, SPREP-1c also inhibits FoxO1 expression through indirect regulation. In $\mathrm{HB}$, it is still unclear whether direct or indirect regulation plays a dominant role. We were unable to carry out a detailed study on this part because of insufficient experimental funds. In the future, we will conduct a series of molecular biology experiments to refine our research.

\section{Conclusion}

FoxO1 could slow down the progress of $\mathrm{HB}$ by inhibiting the fatty acid metabolism while SREBP-1c promotes it. FoxO1 and SREBP-1c have an inhibitory effect on each other. Coordinated regulation of FoxO1 and SREBP-1c facilitated the progression of $\mathrm{HB}$ by regulating fatty acid metabolism in vivo and vitro. These findings provided a theoretical basis for a better understanding of the mechanism of fatty acid metabolism in HB. They helped to develop new targets for the clinical treatment of $\mathrm{HB}$.
Abbreviations
HB:
FASN: $\quad$ Fatty acid synthase
ACC: Acetyl-coenzyme A carboxylase
FoxO1: Forkhead box-O1 
SREBP-1c: Sterol regulatory element-binding protein-1c

ACLY: $\quad$ ATP-citric acid lyase

MAGL: $\quad$ Monoacylglycerol lipase

TG: $\quad$ Triglyceride

FAO: $\quad$ Fatty acid oxidase

Acetyl-CoA: Acetyl-coenzyme A

NEFA: $\quad$ Nonesterified fatty acid.

\section{Data Availability}

The data used to support the findings of this study are available from the corresponding authors upon request.

\section{Conflicts of Interest}

The authors declare that there is no conflict of interest regarding the publication of this article.

\section{Authors' Contributions}

Qin Zhu, Yu Hu, and Hongyan Zai conceptualized the study design. Yu Hu, Hongyan Zai, Wei Jiang, Zhenglin Ou, and Yuanbing Yao collected specimens and experimental data. Hongyan Zai and Wei Jiang did a statistical analysis of the data. Zhenglin $\mathrm{Ou}$ and Yuanbing Yao wrote the first draft of the manuscript.

\section{Acknowledgments}

This work was supported by Xiangya Hospital.

\section{References}

[1] I. Cristóbal, M. Sanz-Álvarez, M. Luque, C. Caramés, F. Rojo, and J. García-Foncillas, "The role of microRNAs in hepatoblastoma tumors," Cancers (Basel), vol. 11, no. 3, p. 409, 2019.

[2] S. R. Musick, M. Smith, A. S. Rouster, and H. M. Babiker, Hepatoblastoma, in StatPearls, StatPearls Publishing Copyright, Treasure Island (FL), 2021.

[3] N. Koundouros and G. Poulogiannis, "Reprogramming of fatty acid metabolism in cancer," British Journal of Cancer, vol. 122, no. 1, pp. 4-22, 2020.

[4] M. Yi, J. Li, S. Chen et al., "Emerging role of lipid metabolism alterations in cancer stem cells," Journal of Experimental \& Clinical Cancer Research, vol. 37, no. 1, p. 118, 2018.

[5] Y. Ma, S. M. Temkin, A. M. Hawkridge et al., "Fatty acid oxidation: an emerging facet of metabolic transformation in cancer," Cancer Letters, vol. 435, pp. 92-100, 2018.

[6] C. W. Fhu and A. Ali, "Fatty acid synthase: an emerging target in cancer," Molecules, vol. 25, no. 17, p. 3935, 2020.

[7] R. K. Yadav, A. S. Chauhan, L. Zhuang, and B. Gan, "FoxO transcription factors in cancer metabolism," Seminars in Cancer Biology, vol. 50, pp. 65-76, 2018.

[8] S. Peng, W. Li, N. Hou, and N. Huang, "A review of FoxO1regulated metabolic diseases and related drug discoveries," Cell, vol. 9, no. 1, 2020.

[9] H. Guan, P. Tan, L. Xie et al., "FOXO1 inhibits osteosarcoma oncogenesis via $\mathrm{Wnt} / \beta$-catenin pathway suppression," Oncogene, vol. 4, no. 9, article e166, 2015.
[10] W. Zhang, S. Patil, B. Chauhan et al., "FoxO1 Regulates Multiple Metabolic Pathways in the Liver:," The Journal of Biological Chemistry, vol. 281, no. 15, pp. 10105-10117, 2006.

[11] M. J. Ang, J. Kim, S. Lee et al., “Transcriptome profiling reveals novel candidate genes related to hippocampal dysfunction in SREBP-1c knockout mice," International Journal of Molecular Sciences, vol. 21, no. 11, p. 4131, 2020.

[12] Y. Zhang, C. Li, C. Hu et al., "Lin28 enhances de novo fatty acid synthesis to promote cancer progression via SREBP-1," EMBO Reports, vol. 20, no. 10, article e48115, 2019.

[13] D. Guo, E. H. Bell, P. Mischel, and A. Chakravarti, "Targeting SREBP-1-driven lipid metabolism to treat cancer," Current Pharmaceutical Design, vol. 20, no. 15, pp. 2619-2626, 2014.

[14] M. Teran-Garcia, A. W. Adamson, G. Yu et al., "Polyunsaturated fatty acid suppression of fatty acid synthase (FASN): evidence for dietary modulation of NF-Y binding to the Fasn promoter by SREBP-1c," The Biochemical Journal, vol. 402, no. 3, pp. 591-600, 2007.

[15] F. Geng, X. Cheng, X. Wu et al., "Inhibition of SOAT1 suppresses glioblastoma growth via blocking SREBP-1-mediated lipogenesis," Clinical Cancer Research, vol. 22, no. 21, pp. 5337-5348, 2016.

[16] Y. Wang, L. Zhang, X. Che, W. Li, Z. Liu, and J. Jiang, "Roles of SIRT1/FoxO1/SREBP-1 in the development of progestin resistance in endometrial cancer," Archives of Gynecology and Obstetrics, vol. 298, no. 5, pp. 961-969, 2018.

[17] Q. He, J. Luo, J. Wu et al., "FoxO1 knockdown promotes fatty acid synthesis via modulating SREBP1 activities in the dairy goat mammary epithelial cells," Journal of Agricultural and Food Chemistry, vol. 68, no. 43, pp. 12067-12078, 2020.

[18] S. E. Woodfield, Y. Shi, R. H. Patel et al., "MDM4 inhibition: a novel therapeutic strategy to reactivate p53 in hepatoblastoma," Scientific Reports, vol. 11, no. 1, p. 2967, 2021.

[19] Z. Luo and P. Cao, "Long noncoding RNA PVT1 promotes hepatoblastoma cell proliferation through activating STAT3," Cancer Management and Research, vol. Volume 11, pp. 8517-8527, 2019.

[20] J. S. Wu, S. R. Sheng, X. H. Liang, and Y. L. Tang, "The role of tumor microenvironment in collective tumor cell invasion," Future Oncology, vol. 13, no. 11, pp. 991-1002, 2017.

[21] D. V. Pham, N. Tilija Pun, and P. H. Park, "Autophagy activation and SREBP-1 induction contribute to fatty acid metabolic reprogramming by leptin in breast cancer cells," Molecular Oncology, vol. 15, no. 2, pp. 657-678, 2021.

[22] M. Sciacovelli and C. Frezza, "Metabolic reprogramming and epithelial-to-mesenchymal transition in cancer," The FEBS Journal, vol. 284, no. 19, pp. 3132-3144, 2017.

[23] Q. Sun, X. Yu, C. Peng et al., "Activation of SREBP-1c alters lipogenesis and promotes tumor growth and metastasis in gastric cancer," Biomedicine \& Pharmacotherapy, vol. 128, article 110274, 2020.

[24] J. Li, Q. Huang, X. Long et al., “CD147 reprograms fatty acid metabolism in hepatocellular carcinoma cells through Akt/mTOR/SREBP1c and P38/PPAR $\alpha$ pathways," Journal of Hepatology, vol. 63, no. 6, pp. 1378-1389, 2015.

[25] Y. C. Chae, J. Y. Kim, J. W. Park et al., "FOXO1 degradation via G9a-mediated methylation promotes cell proliferation in colon cancer," Nucleic Acids Research, vol. 47, no. 4, pp. 1692-1705, 2019.

[26] Y. Wang, Z. Lyu, Y. Qin et al., "FOXO1 promotes tumor progression by increased M2 macrophage infiltration in 
esophageal squamous cell carcinoma," Theranostics, vol. 10, no. 25, pp. 11535-11548, 2020.

[27] Y. Liu, Y. Yang, L. Li et al., "LncRNA SNHG1 enhances cell proliferation, migration, and invasion in cervical cancer," Biochemistry and Cell Biology, vol. 96, no. 1, pp. 38-43, 2018.

[28] X. Zhu, H. Bian, L. Wang et al., "Berberine attenuates nonalcoholic hepatic steatosis through the AMPK- SREBP-1c-SCD1 pathway," Free Radical Biology \& Medicine, vol. 141, pp. 192-204, 2019.

[29] T. Tao, Q. Su, S. Xu et al., "Down-regulation of PKM2 decreases FASN expression in bladder cancer cells through AKT/mTOR/SREBP-1c axis," Journal of Cellular Physiology, vol. 234, no. 3, pp. 3088-3104, 2019.

[30] F. Ying, H. Liu, E. H. Ching Tang et al., "Prostaglandin E receptor subtype 4 protects against diabetic cardiomyopathy by modulating cardiac fatty acid metabolism via FOXO1/CD36 signalling," Biochemical and Biophysical Research Communications, vol. 548, pp. 196-203, 2021.

[31] M. G. Vander Heiden, L. C. Cantley, and C. B. Thompson, "Understanding the Warburg effect: the metabolic requirements of cell proliferation," Science, vol. 324, no. 5930, pp. 1029-1033, 2009.

[32] A. Carracedo, L. C. Cantley, and P. P. Pandolfi, "Cancer metabolism: fatty acid oxidation in the limelight," Nature Reviews Cancer, vol. 13, no. 4, pp. 227-232, 2013.

[33] L. S. Pike, A. L. Smift, N. J. Croteau, D. A. Ferrick, and M. Wu, "Inhibition of fatty acid oxidation by etomoxir impairs $\mathrm{NADPH}$ production and increases reactive oxygen species resulting in ATP depletion and cell death in human glioblastoma cells," Biochimica et Biophysica Acta, vol. 1807, no. 6, pp. 726-734, 2011.

[34] A. Vazquez, J. J. Kamphorst, E. K. Markert, Z. T. Schug, S. Tardito, and E. Gottlieb, "Cancer metabolism at a glance," Journal of Cell Science, vol. 129, no. 18, pp. 3367-3373, 2016.

[35] A. Carrer, S. Trefely, S. Zhao et al., "Acetyl-CoA metabolism supports multistep pancreatic tumorigenesis," Cancer Discovery, vol. 9, no. 3, pp. 416-435, 2019.

[36] I. S. Senatorov and N. H. Moniri, "The role of free-fatty acid receptor-4 (FFA4) in human cancers and cancer cell lines," Biochemical Pharmacology, vol. 150, pp. 170-180, 2018.

[37] P. Li, M. Lu, J. Shi et al., "Lung mesenchymal cells elicit lipid storage in neutrophils that fuel breast cancer lung metastasis," Nature Immunology, vol. 21, no. 11, pp. 1444-1455, 2020.

[38] Y. Li and S. Wu, "Epigallocatechin gallate suppresses hepatic cholesterol synthesis by targeting SREBP-2 through SIRT1/FOXO1 signaling pathway," Molecular and Cellular Biochemistry, vol. 448, no. 1-2, pp. 175-185, 2018.

[39] S. K. Bose, H. Kim, K. Meyer, N. Wolins, N. O. Davidson, and R. Ray, "Forkhead box transcription factor regulation and lipid accumulation by hepatitis C virus," Journal of Virology, vol. 88, no. 8, pp. 4195-4203, 2014.

[40] X. Deng, W. Zhang, I. S. O-Sullivan et al., "FoxO1 Inhibits Sterol Regulatory Element-binding Protein-1c (SREBP-1c) Gene Expression via Transcription Factors Sp1 and SREBP-1c," The Journal of Biological Chemistry, vol. 287, no. 24, pp. 20132-20143, 2012.

[41] M. P. Sajan, M. C. Lee, F. Foufelle, J. Sajan, C. Cleland, and R. V. Farese, "Coordinated regulation of hepatic FoxO1, PGC- $1 \alpha$ and SREBP-1c facilitates insulin action and resistance," Cellular Signalling, vol. 43, pp. 62-70, 2018. 